\title{
Evolución de la distribución de las reservas de hidrocarburos de las Provincias Petroleras Mexicanas
}

\author{
Evolution of the distribution of the hydrocarbon reserves of the Mexican Petroleum \\ Provinces
}

\author{
Diana Hernández Martínez ${ }^{\mathrm{a}}$, Luca Ferraria \\ ${ }^{a}$ Centro de Geociencias, Universidad Nacional Autónoma de México, Campus Juriquilla, Querétaro, 76230, México
}

\begin{abstract}
Resumen
En el presente trabajo se presenta, a través de diferentes mapas, la información concerniente a 1,110 campos de petróleo y gas que se ha podido recopilar integrando diferentes bases de datos institucionales. La información recopilada contiene un $42 \%$ adicional de pozos con respecto al catastro petrolero de la Comisión Nacional de Hidrocarburos. Con la información de esta nueva base de datos y los datos de reservas certificadas al 1 de enero de 2016, se obtuvieron 18 mapas que permiten visualizar a nivel de Provincia Petrolera y Geológica la ubicación geográfica de los campos descubiertos en México, la distribución de la producción acumulada de hidrocarburos, las reservas remanentes y el volumen original para crudo y gas natural en su clasificación 1P, 2P y 3P. Mediante estos mapas es posible apreciar visualmente las áreas con mayor exploración y desarrollo así como la ubicación de los campos con mayor volumen original. Los mapas nos permiten entender la evolución de la explotación de los yacimientos y su estado actual así como identificar las áreas remanentes para exploración y producción.
\end{abstract}

Palabras clave: México; Producción de hidrocarburos; Distribución original de hidrocarburos; Distribución de reservas.

\begin{abstract}
In the present work, information concerning 1,110 oil and gas fields compiled by integrating different databases is presented through different maps. This new database contains an additional $42 \%$ of data with respect to the oil land registry of the National Hydrocarbons Commission. With the information of database and the data of reserves certified as of January 1, 2016, we produced 18 maps that visualize the geographical location of the fields discovered in Mexico, the distribution of cumulative hydrocarbon production, the remaining reserves, and the original volume of oil and gas in its $1 \mathrm{P}, 2 \mathrm{P}$ and $3 \mathrm{P}$ classification. Through these maps it is possible to identify the areas with more intense exploration and development and the location of the fields with the largest original volume. The maps show the evolution of the exploitation of the fields and their current states; they also identify the remaining areas for exploration and production.
\end{abstract}

Keywords: Mexico; Hydrocarbon production; Original distribution of hydrocarbon; Distribution of reserves.

\section{Introducción}

El petróleo en el mundo se encuentra estrechamente vinculado a importantes acontecimientos sociales, políticos y econó-

\footnotetext{
(C) Diana Hernández Martínez and Luca Ferrari. Published by Terra Digitalis.

This is an Open Access article distributed under the terms of the Creative Commons Attribution License (https://creativecommons.org/licenses/by-nc-sa/4.0/), which permits non-commencial sharing of the work and adaptions, provided the original work is properly cited and the new creations are licensed under identical terms.

*E-mail address: luca@unam.mx
}

micos (Bentham, 2014). La historia de México y el desarrollo de la Nación se han trazado en relación a las reservas y aprovechamiento de los recursos naturales y minerales, entre ellos los hidrocarburos (Álvarez de la Borda, 2006). En la segunda mitad del siglo XX, México adquirió renombre y un lugar en el escenario mundial por su riqueza energética basada en los hidrocarburos, la cual le permitió satisfacer la demanda interna de petróleo y exportar excedentes al resto del mundo (INEGI, 2015). La renta petrolera llegó a representar entre el $8 \%$ y $10 \%$ del PIB entre 1979 y 1985 , para caer por debajo del $3 \%$ en la década de los 90; sin embargo entre 2005 y 2012 volvió a reba- 
sar el $5 \%$ (Banco Mundial, 2016).

Las reservas probadas de hidrocarburos se consideran el indicador petrolero de mayor importancia, ya que estiman el volumen de hidrocarburos que puede ser producido. Las evaluaciones de recursos estiman las cantidades totales en acumulaciones conocidas o aún por descubrir de acuerdo a un rango de incertidumbre (SPE et al., 2007).

Desde 1980 hasta la primera mitad de la década de los 90's, México se mantuvo entre los 10 países con mayor reserva de petróleo a nivel mundial (BP, 2016). De acuerdo a la información publicada por la Comisión Nacional de Hidrocarburos (CNH), las reservas totales de petróleo han declinado de 2004 a 2015 a una tasa anual del $5 \%$.

Los volúmenes certificados de reservas 1P en 2016 (Tabla 1) permiten continuar la producción de crudo al ritmo actual por sólo 9.2 años y de gas por 6.3 años $(\mathrm{CNH}, 2017$; HernándezMartínez, 2017). La declinación natural de los yacimientos y la baja tasa de incorporación de reservas llevan a México en 2016 a ocupar el lugar 18 y 35 en cuanto a volumen de reservas probadas de crudo y gas, respectivamente. Actualmente el país posee el $0.6 \%$ de las reservas de crudo y $0.2 \%$ de las reservas de gas del mundo (BP, 2016).

Con el propósito de apreciar la distribución de los recursos petroleros de México y su evolución histórica se elaboraron 18 mapas de interpolación, los cuales permiten visualizar la distribución geográfica de los campos petroleros, sus reservas iniciales y su reservas remanentes.

\section{Métodos}

Los mapas de distribución de las reservas de hidrocarburos y producción acumulada son resultado de la integración de datos de la literatura y mapas del inventario de campos petroleros descubiertos en México, y su procesamiento mediante interpolación de datos de las Reservas de Hidrocarburos certificadas al 1 de enero de 2016 (CNH, 2017a).

\subsection{Base de datos de Campos Petroleros}

La información de los campos petroleros se obtuvo a partir de la revisión y compilación en una base de datos de la información disponible en publicaciones de Petróleos Mexicanos (PEMEX) tales como: Memoria de Labores (1965-2013),
Anuario Estadístico (1977-2015), Las Reservas de Hidrocarburos de México (2002-2015), Informe Anual (2002-2014) y Base de Datos Institucional de PEMEX (BDI). Asimismo se consultó la documentación e información publicada por la CNH y la Secretaría de Energía (SENER), el Sistema de Información Energética (SIE), el Sistema Nacional de Información de Hidrocarburos (SNIH) y el Centro Nacional de Información de Hidrocarburos (CNIH), así como diversos libros, revistas, artículos, tesis y mapas de divulgación científica. Se accedió a información de PEMEX, CNH y SENER que no se encontraba disponible públicamente mediante un total de 14 solicitudes de información a través de la Plataforma Nacional de Transparencia del Instituto Nacional de Transparencia, Acceso a la Información y protección de Datos Personales (INAI).

La información concerniente a la ubicación de los campos fue georreferenciada e integrada en un sistema de información geográfica. Como base para la ubicación de diversos campos de hidrocarburos se utilizó el conjunto de shapefiles de campos petroleros emitido por la $\mathrm{CNH}$ en el CNIH. Es importante señalar que la $\mathrm{CNH}$ especifica que los polígonos sólo son indicativos en superficie, debido a que en subsuelo los límites de los campos y yacimientos pueden variar. De hecho en algunos casos los límites tienen trazos geométricos regulares que probablemente corresponden a la configuración de bloques que delimitan las áreas susceptibles de adjudicarse a través de asignaciones, más que a la posible configuración geológica del yacimiento (DOF, 2014). En estos casos se realizó una modificación de la configuración de los polígonos basada principalmente en la distribución histórica de los pozos y la georreferenciación de mapas publicados por PEMEX. Las bases de datos de SENER y CNH contienen únicamente un inventario de 772 campos petroleros, por lo que otros 338 campos que no se encontraron dentro de los shapefiles proporcionados por la $\mathrm{CNH}$ fueron georreferenciados de mapas de CNH, PEMEX y SENER, y ajustados con la localización de pozos perforados.

\subsection{Datos de Reservas de Hidrocarburos al 1 de enero 2016}

La CNH (2012) califica las reservas de hidrocarburos como "los indicadores más importantes de información que los gobiernos, el sector financiero y las empresas petroleras requieren para definir las acciones y asegurar la sustentabilidad de largo plazo". Las reservas de hidrocarburos son cantidades estimadas de petróleo que pueden ser recuperadas comercialmente y

Tabla 1: Reservas de Hidrocarburos Certificadas al 1 de enero de 2016

Table 1: Certified Hydrocarbon Reserves as of January 1, 2016

\begin{tabular}{|c|c|c|c|c|c|c|c|c|c|c|c|c|c|}
\hline \multicolumn{2}{|c|}{ Volumen original 3P } & \multicolumn{9}{|c|}{ Reservas remanentes de hidrocarburos } & \multicolumn{3}{|c|}{ Producción acumulada } \\
\hline $\begin{array}{l}\text { Crudo } \\
\text { (mmb*) }\end{array}$ & $\begin{array}{c}\text { Gas natural } \\
\left(\text { mmmpc }^{\#}\right)\end{array}$ & & $\begin{array}{c}\text { PCE } \\
\text { nmbpce }\end{array}$ & & & $\begin{array}{l}\text { Crudo } \\
\text { (mmb) }\end{array}$ & & & $\begin{array}{l}\text { as natu } \\
\text { mmmp }\end{array}$ & & $\begin{array}{c}\text { PCE } \\
\text { (mmbpce) }\end{array}$ & $\begin{array}{l}\text { Crudo } \\
\text { (mmb) }\end{array}$ & $\begin{array}{l}\text { Gas natural } \\
\text { (mmmpc) }\end{array}$ \\
\hline & & $1 \mathrm{P}$ & $2 \mathrm{P}$ & $3 \mathrm{P}$ & $1 \mathrm{P}$ & $2 \mathrm{P}$ & $3 \mathrm{P}$ & $1 \mathrm{P}$ & $2 \mathrm{P}$ & $3 \mathrm{P}$ & & & \\
\hline
\end{tabular}

Fuente: Comisión Nacional de Hidrocarburos.

Notas: PCE: Petróleo Crudo Equivalente; $\mathbf{m m b}{ }^{*}$ : Millones de barriles; $\boldsymbol{m m \boldsymbol { m }} \boldsymbol{c}^{\#}$ : Miles de millones de pies cúbicos; $\boldsymbol{m m b} \boldsymbol{m} \boldsymbol{e}^{+}$: Millones de barriles de petróleo crudo equivalente 
son clasificadas de acuerdo con el nivel de certeza asociado con las estimaciones (SPE et al., 2007). De acuerdo a la clasificación internacional (SPE et al., 2007) las reservas 1P (probadas) corresponden a volúmenes de crudo y gas natural que tienen un alto potencial ( $90 \%$ de probabilidad) de recuperación en un tiempo determinado. Dicha probabilidad depende tanto de consideraciones geológico-técnicas como del costo de extracción. Las reservas $2 \mathrm{P}$ corresponden a las reservas probadas más las probables, que en conjunto tienen al menos un $50 \%$ de probabilidad de ser extraídas. Finalmente las reservas 3P son la suma de las reservas probadas, probables y posibles, que tienen un $10 \%$ de probabilidad de ser extraídas. El volumen original es la cantidad de hidrocarburo que se estima existe originalmente en el yacimiento, y está confinado por límites geológicos y de características de los fluidos, pudiéndose expresar tanto a condiciones de yacimiento como a condiciones de superficie (PEMEX, 2010).

Para este trabajo se utilizó la base de datos de los reportes estadísticos de recursos y reservas en su clasificación por campos publicada por la Comisión Nacional de Hidrocarburos (CNH, 2017a). La información incluye la revisión a las reservas $2 \mathrm{P}$ y $3 \mathrm{P}$ en petróleo crudo equivalente, crudo y gas natural realizada al campo Akal (CNH, 2017b), campo principal del complejo Cantarell. Los datos de reservas de hidrocarburos incluyen las estimaciones de petróleo crudo equivalente, crudo y gas natural correspondiente a Producción Acumulada, Volumen Original y Reservas Remanentes en sus tres clasificaciones 1P, $2 \mathrm{P}$ y $3 \mathrm{P}$.

\subsection{Interpolación de Datos}

Para la elaboración de los modelos de interpolación se utilizó el método de distancia inversa ponderada (IDW, por sus siglas en inglés). El método IDW realiza las estimaciones por influencia de la proximidad o distancia de los datos, en la que se asigna una ponderación mayor a los puntos más cercanos, que a aquellos que se encuentran más alejados (Burrough y McDonnell, 1998; Longley, 2005; Xu et al., 2012).

La interpolación por IDW es adecuada para representar la distribución de las reservas de hidrocarburos debido a que es congruente con el método de clasificación de reservas, donde la incertidumbre se incrementa con la distancia de un pozo y por ende la clasificación de los recursos cambia, como se muestra en la Figura 1 (Barker, 2008; SPE et al., 2011).

\section{Resultados}

Con la compilación de información de campos petroleros descubiertos en México se incrementó el catastro a 1,110 campos petroleros. Se obtuvieron 18 mapas que permiten visualizar a nivel de Provincia Petrolera y Geológica la distribución geográfica en los campos descubiertos en México, la distribución de la producción acumulada de hidrocarburos, las reservas remanentes y volumen original en su clasificación $1 \mathrm{P}, 2 \mathrm{P}$ y $3 \mathrm{P}$ de petróleo crudo equivalente, crudo y gas natural, certificados al 1 de enero de 2016. Con estos mapas es posible determinar visualmente la ubicación de los campos con mayor volumen original descubierto así como la historia y potencial de producción.

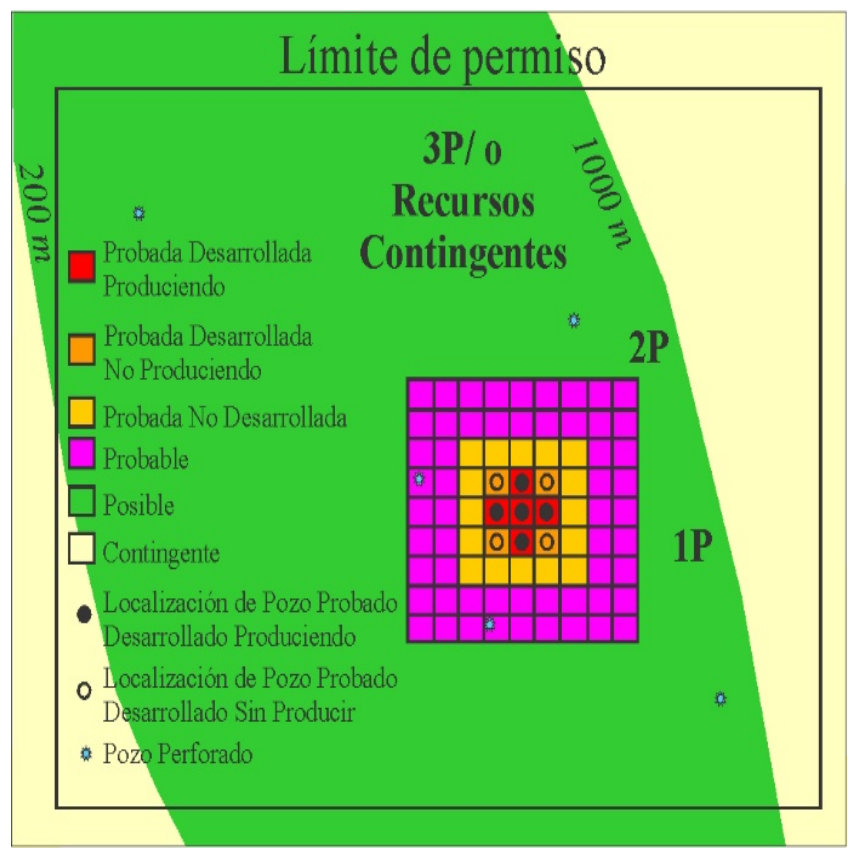

Figura 1: Variación de la clasificación de recursos petroleros con la distancia. Tomada de Barker, 2008.

Figure 1: Variation of the oil resources classification with the distance, after Barker (2008).

Los mapas de volumen original $1 \mathrm{P}$ de crudo muestran que en buena medida la riqueza petrolera de México estaba concentrada en la provincia petrolera de las Cuencas del Sureste, tanto en su porción terrestre como mar adentro de la Sonda de Campeche (Figura 2). La segunda concentración importante corresponde a la parte norte de la provincia Tampico-Misantla y en menor medida a su parte central con la llamada "Faja de Oro" (Figura 2). En los mapas del volumen original 2P, aparece una concentración en el área del llamado paleocanal de Chicontepec (Figura 3), que por sus características geológicas (baja permeabilidad y estratos discontinuos) tiene una baja tasa de recuperación y altos costos de extracción. Finalmente, considerando el volumen original $3 \mathrm{P}$, se añade el área de aguas profundas del Cinturón Plegado Perdido, cerca de la frontera con Estados Unidos (Figura 4). La alta concentración de hidrocarburos en las Provincias Petroleras de Cuencas del Sureste y Tampico Misantla las convierte en las principales productoras de hidrocarburos como se observa en la Figura 5. Por las décadas de producción de hidrocarburos, declinación de los yacimientos y baja restitución, actualmente observamos las reservas $1 \mathrm{P}$ de crudo concentradas principalmente en el área marina de la Sonda de Campeche (Figura 6), considerando las reservas 2P y 3P, se adicionan las zonas de Chicontepec en la Provincia Tampico Misantla y Área Perdido en el Golfo Profundo, respectivamente (Figuras 7 y 8 ). 
Figura 2: Distribución de Volumen original 1P de Crudo

Figure 2: Distribution of the original 1P volume of oil

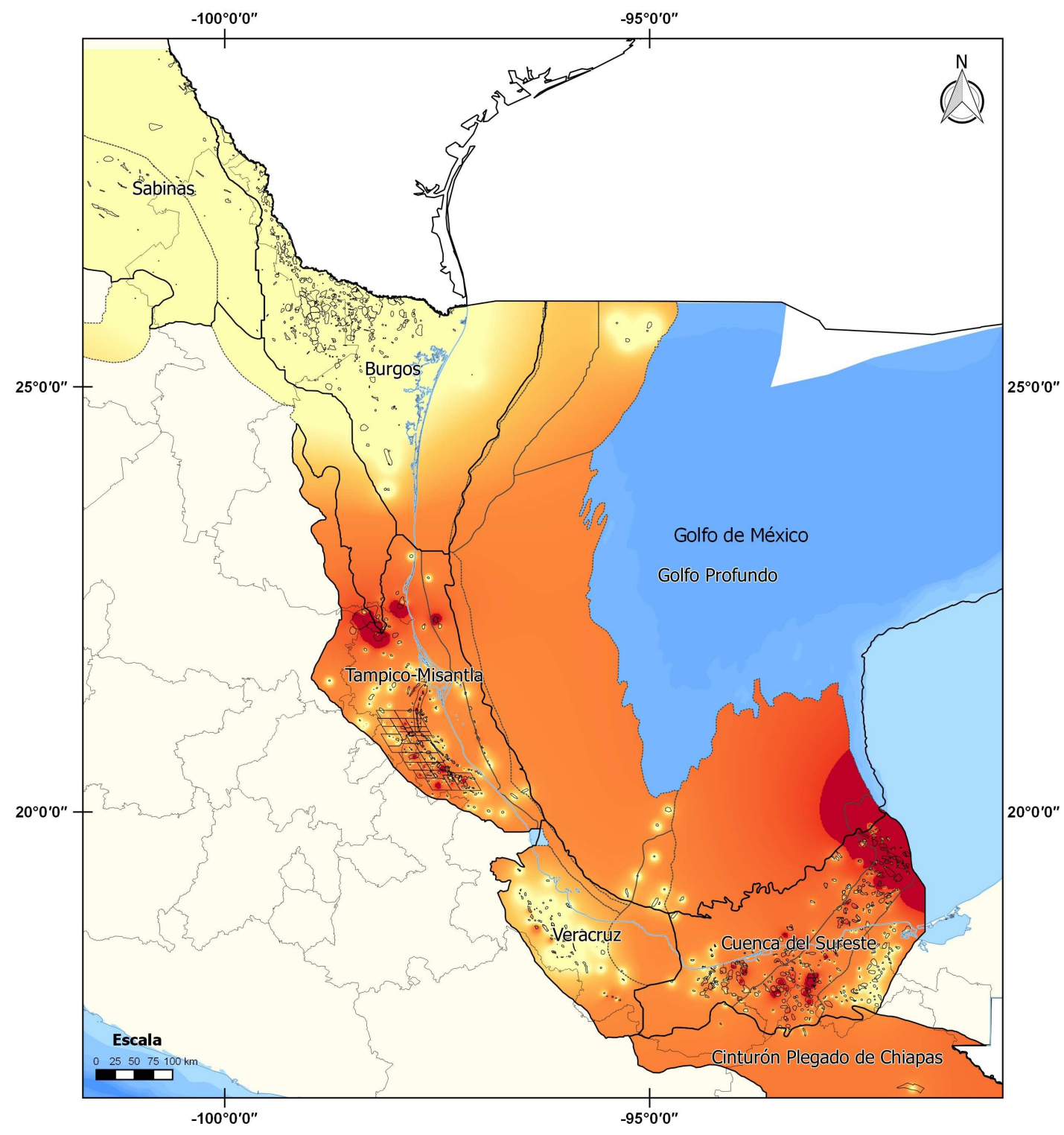

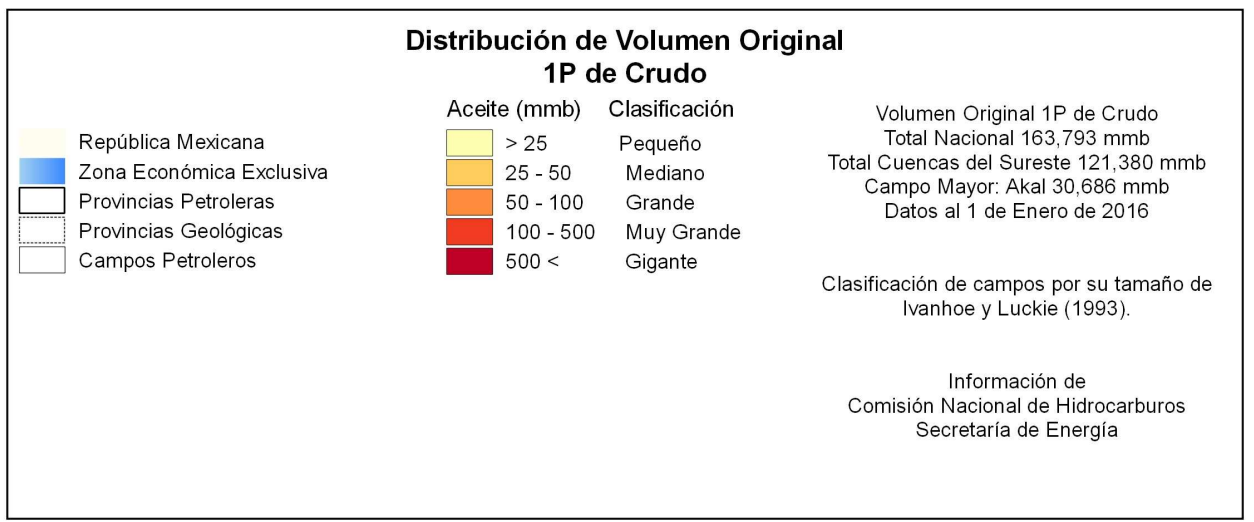


Figura 3: Distribución de Volumen original 2P de Crudo

Figure 3: Distribution of the original $2 \mathrm{P}$ volume of oil

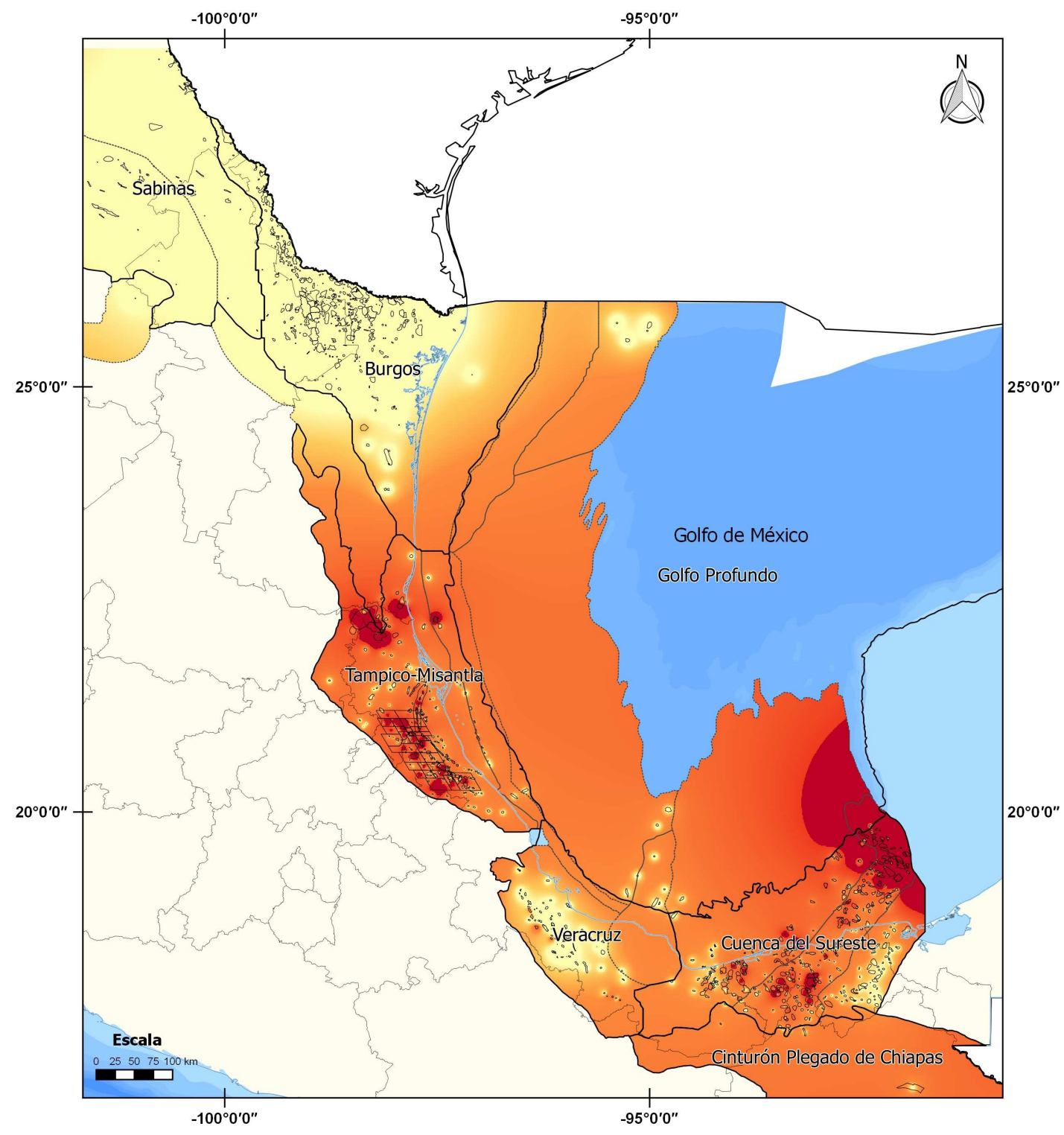

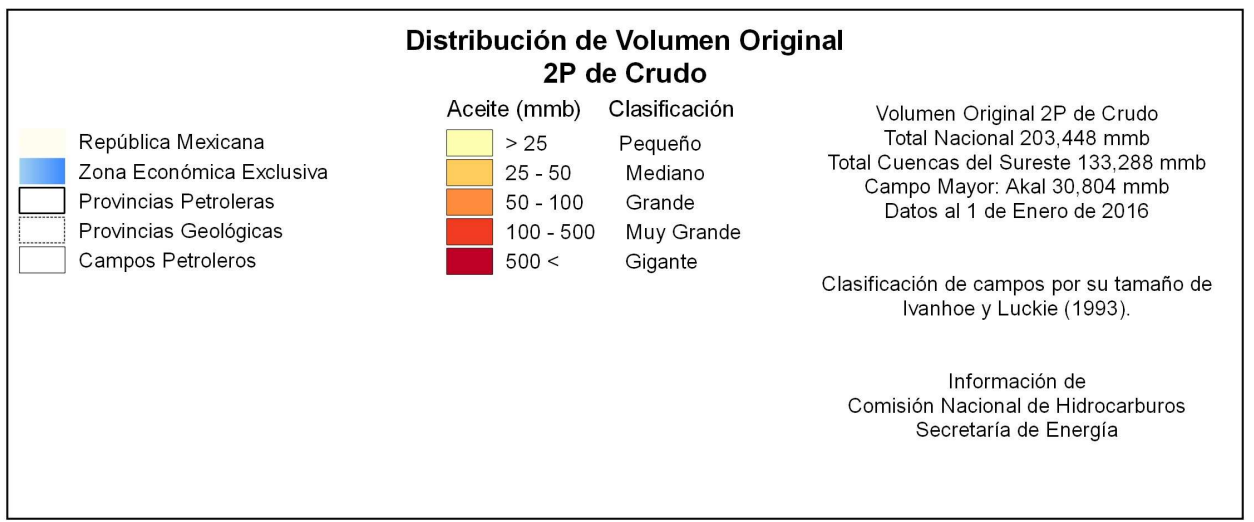


Figura 4: Distribución de Volumen original 3P de Crudo

Figure 4: Distribution of the original 3P volume of oil

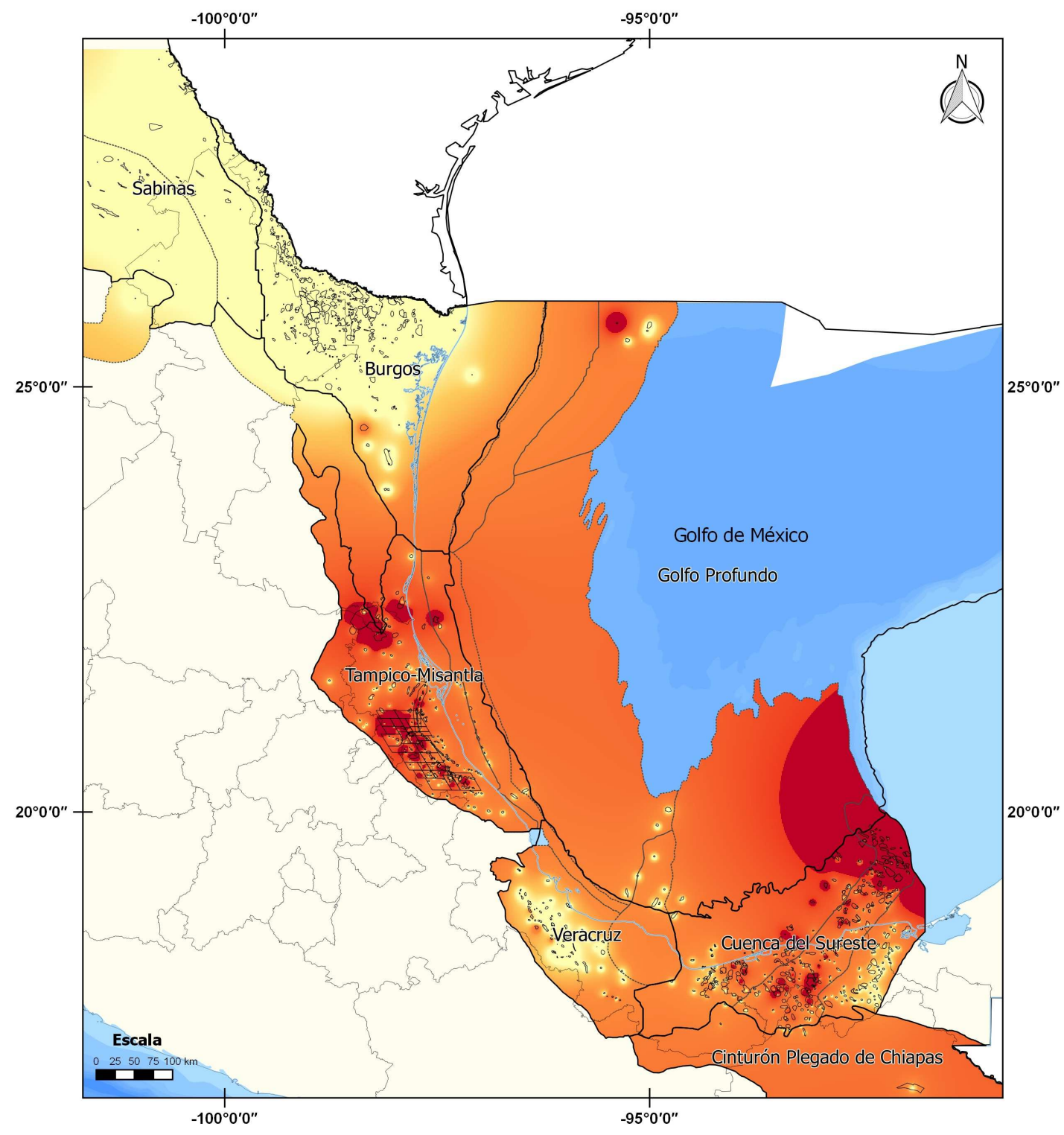

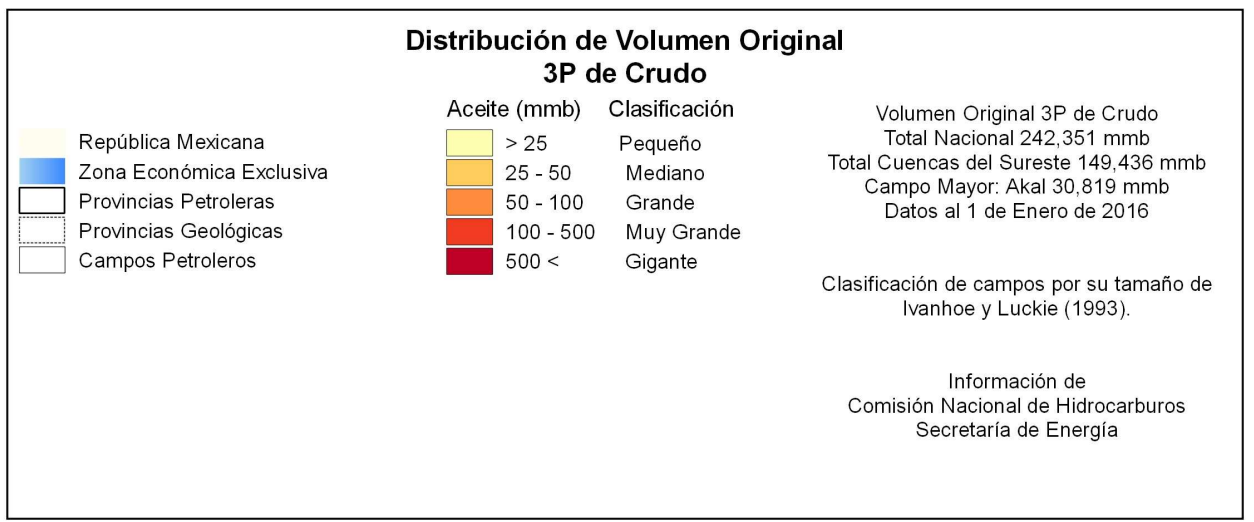


Figura 5: Distribución de la producción acumulada de crudo

Figura 5 : Distribution of cumulative oil production

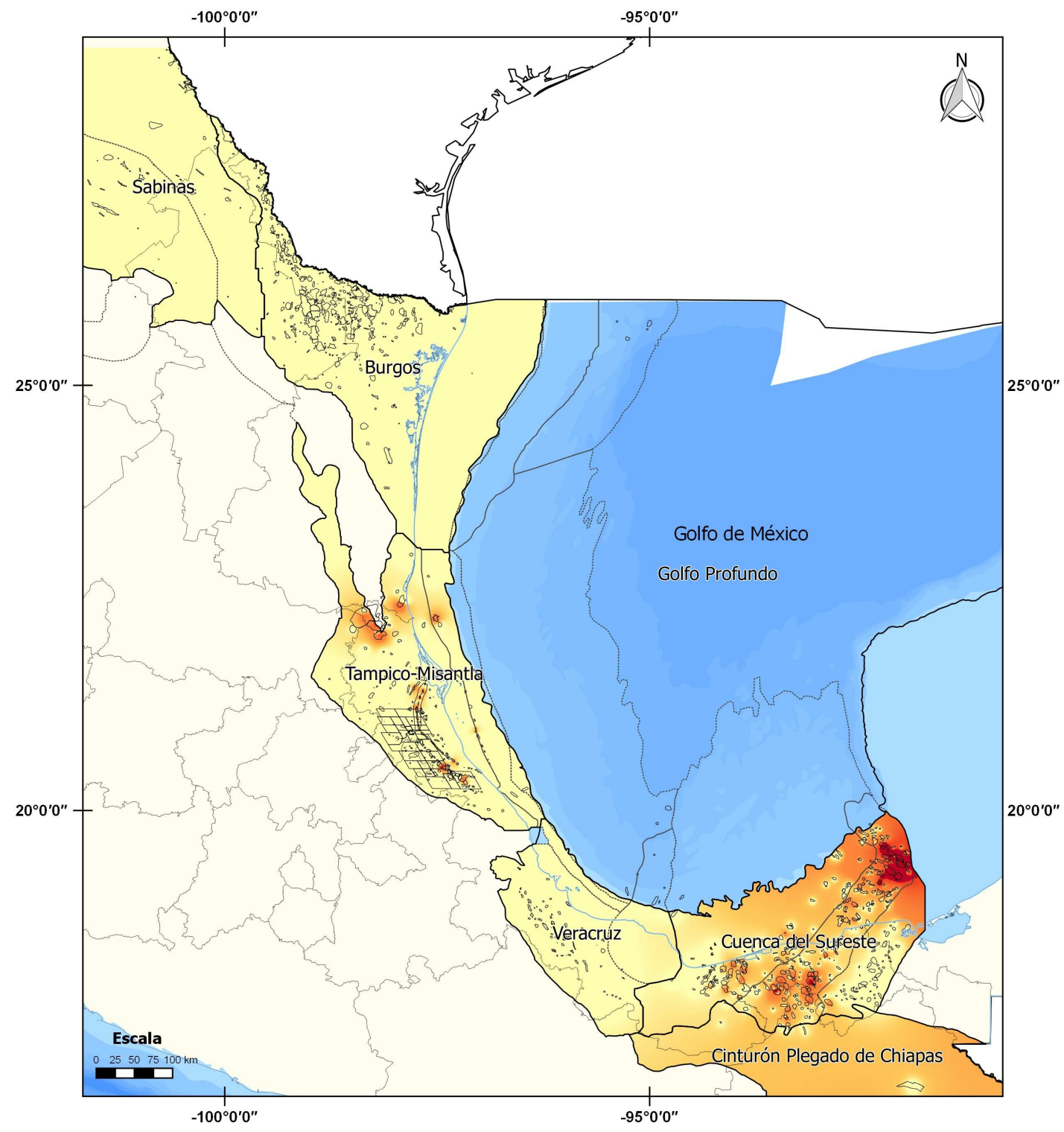

\begin{tabular}{|c|c|c|c|}
\hline \multicolumn{4}{|c|}{ Distribución de la Producción Acumulada de Crudo } \\
\hline $\begin{array}{l}\text { República Mexicana } \\
\text { Zona Económica Exclusiva } \\
\text { Provincias Petroleras } \\
\text { Provincias Geológicas } \\
\text { Campos Petroleros }\end{array}$ & 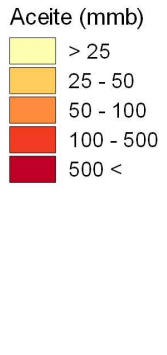 & $\begin{array}{l}\text { Clasificación } \\
\text { Pequeño } \\
\text { Mediano } \\
\text { Grande } \\
\text { Muy Grande } \\
\text { Gigante }\end{array}$ & $\begin{array}{c}\text { Producción Acumulada de Crudo } \\
\text { Total Nacional } 43,262 \mathrm{mmb} \\
\text { Total Cuencas del Sureste } 37,287 \mathrm{mmb} \\
\text { Campo Mayor: Akal 12,890 mmb } \\
\text { Datos al 1 de Enero de } 2016 \\
\text { Clasificación de campos por su tamaño de } \\
\text { Ivanhoe y Luckie (1993). } \\
\text { Información de } \\
\text { Comisión Nacional de Hidrocarburos } \\
\text { Secretaría de Energía }\end{array}$ \\
\hline
\end{tabular}


Figura 6: Distribución de reservas 1P de crudo

Figure 6: Distribution of 1P reserve of oil

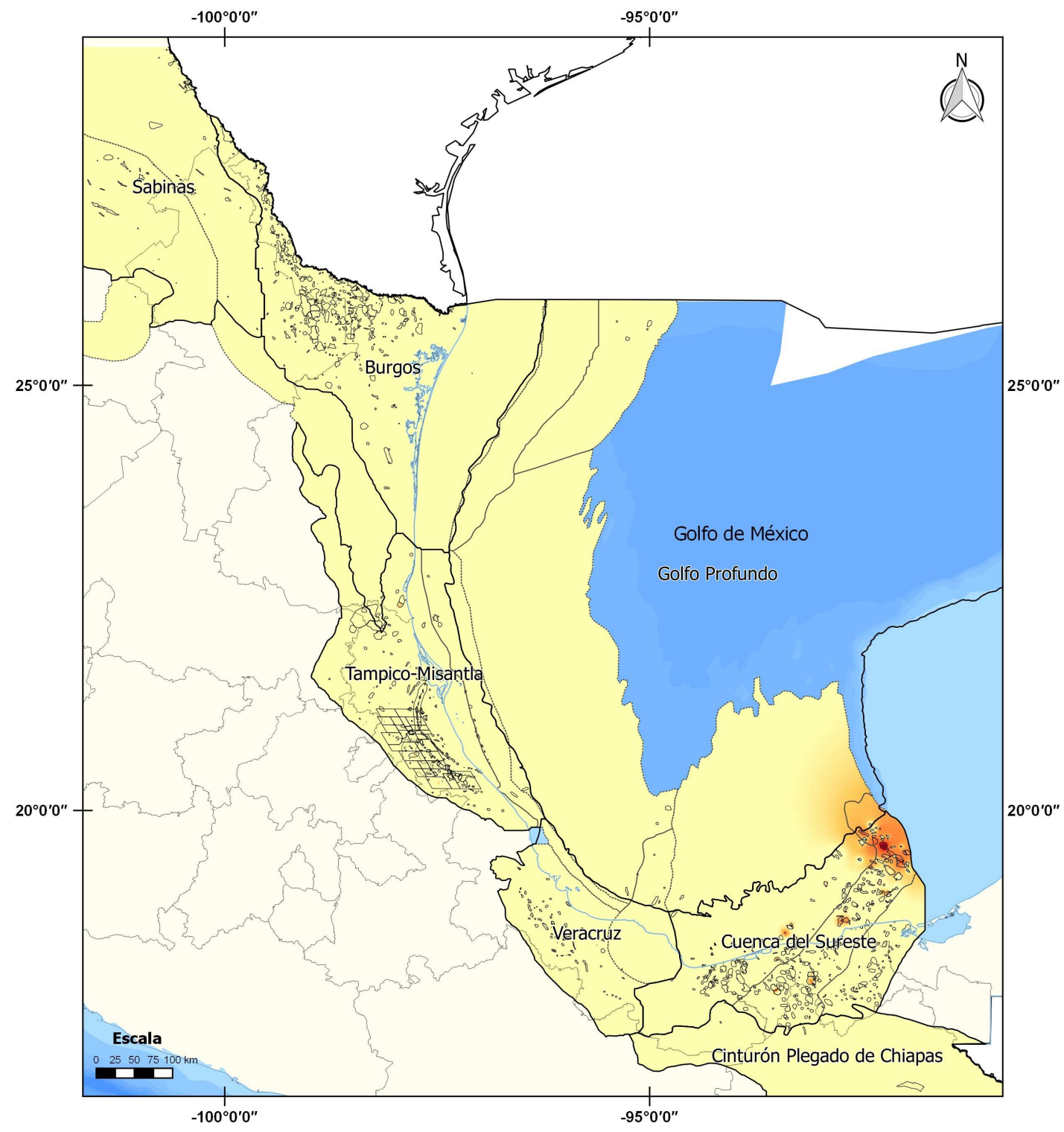

\begin{tabular}{|c|c|c|c|}
\hline \multicolumn{4}{|c|}{ Distribución de Reservas 1P de Crudo } \\
\hline 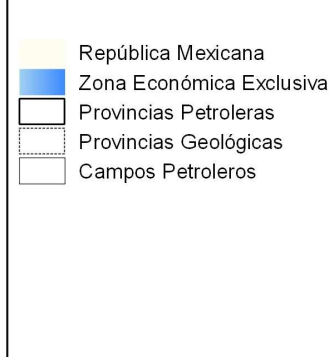 & 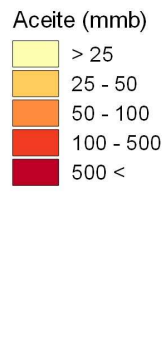 & $\begin{array}{l}\text { Clasificación } \\
\text { Pequeño } \\
\text { Mediano } \\
\text { Grande } \\
\text { Muy Grande } \\
\text { Gigante }\end{array}$ & $\begin{array}{c}\text { Reservas } 1 \mathrm{P} \text { de Crudo } \\
\text { Total Nacional } 7,641 \text { mmbpce } \\
\text { Total Cuencas del Sureste 6,881 mmbpce } \\
\text { Campo Mayor: Maloob 1,473 mmbpce } \\
\text { Datos al } 1 \text { de Enero de } 2016 \\
\text { Clasificación de campos por su tamaño de } \\
\text { Ivanhoe y Luckie (1993). } \\
\text { Información de } \\
\text { Comisión Nacional de Hidrocarburos } \\
\text { Secretaría de Energía }\end{array}$ \\
\hline
\end{tabular}


Figura 7: Distribución de reservas $2 \mathrm{P}$ de crudo

Figure 7: Distribution of $2 \mathrm{P}$ reserve of oil

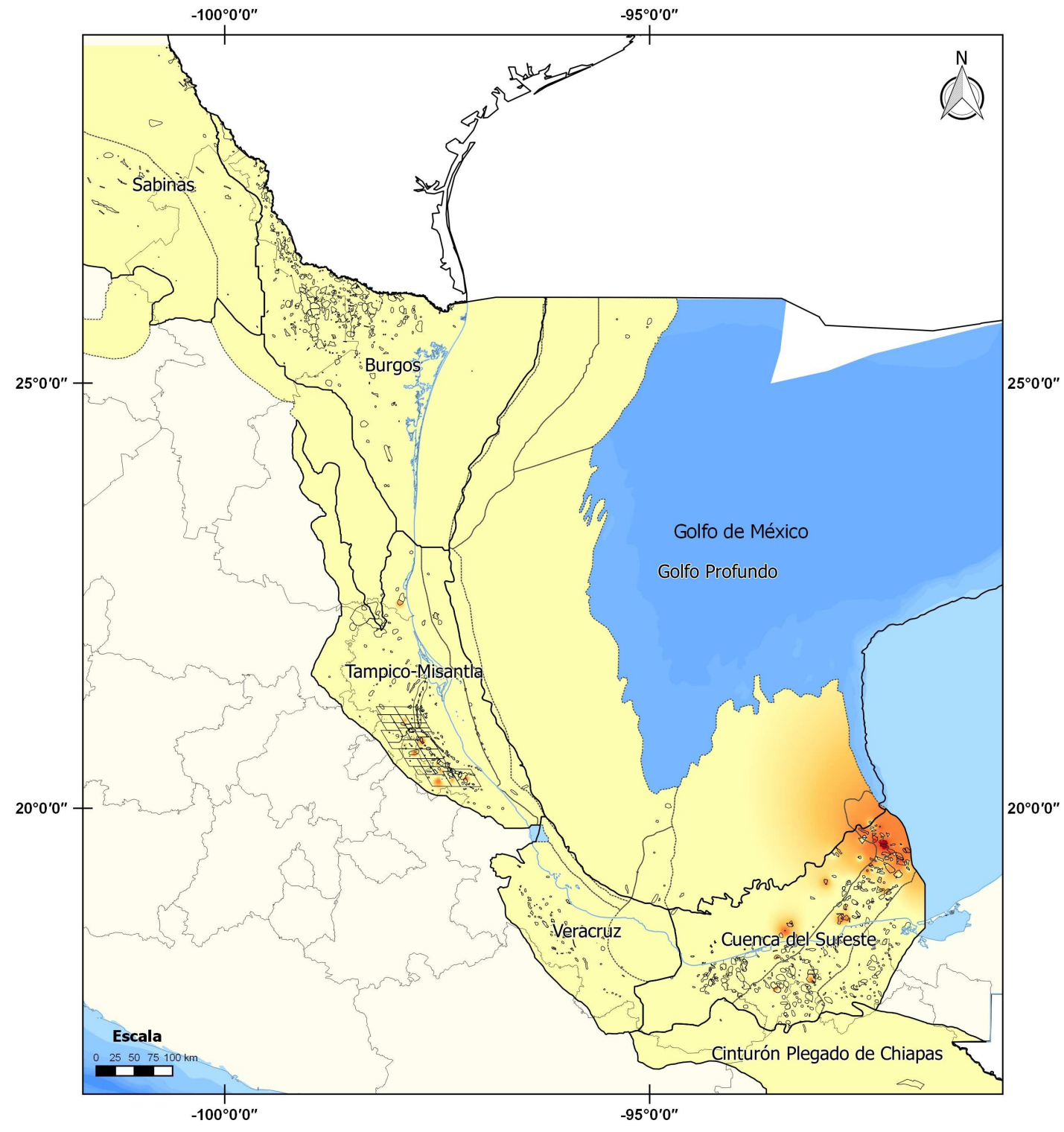

\begin{tabular}{|c|c|c|c|}
\hline \multicolumn{4}{|c|}{ Distribución de Reservas 2P de Crudo } \\
\hline $\begin{array}{l}\text { República Mexicana } \\
\text { Zona Económica Exclusiva } \\
\text { Provincias Petroleras } \\
\text { Provincias Geológicas } \\
\text { Campos Petroleros }\end{array}$ & 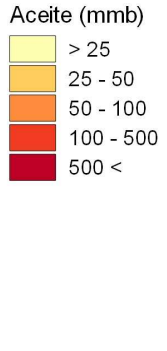 & $\begin{array}{l}\text { Clasificación } \\
\text { Pequeño } \\
\text { Mediano } \\
\text { Grande } \\
\text { Muy Grande } \\
\text { Gigante }\end{array}$ & $\begin{array}{c}\text { Reservas } 2 \mathrm{P} \text { de Crudo } \\
\text { Total Nacional } 13,273 \text { mmbpce } \\
\text { Total Cuencas del Sureste } 10,549 \text { mmbpce } \\
\text { Campo Mayor: Akal } 1,735 \text { mmbpce } \\
\text { Datos al } 1 \text { de Enero de } 2016 \\
\text { Clasificación de campos por su tamaño de } \\
\text { Ivanhoe y Luckie (1993). } \\
\text { Información de } \\
\text { Comisión Nacional de Hidrocarburos } \\
\text { Secretaría de Energía }\end{array}$ \\
\hline
\end{tabular}


Figura 8: Distribución de reservas 3P de crudo

Figure 8: Distribution of 3P reserve of oil

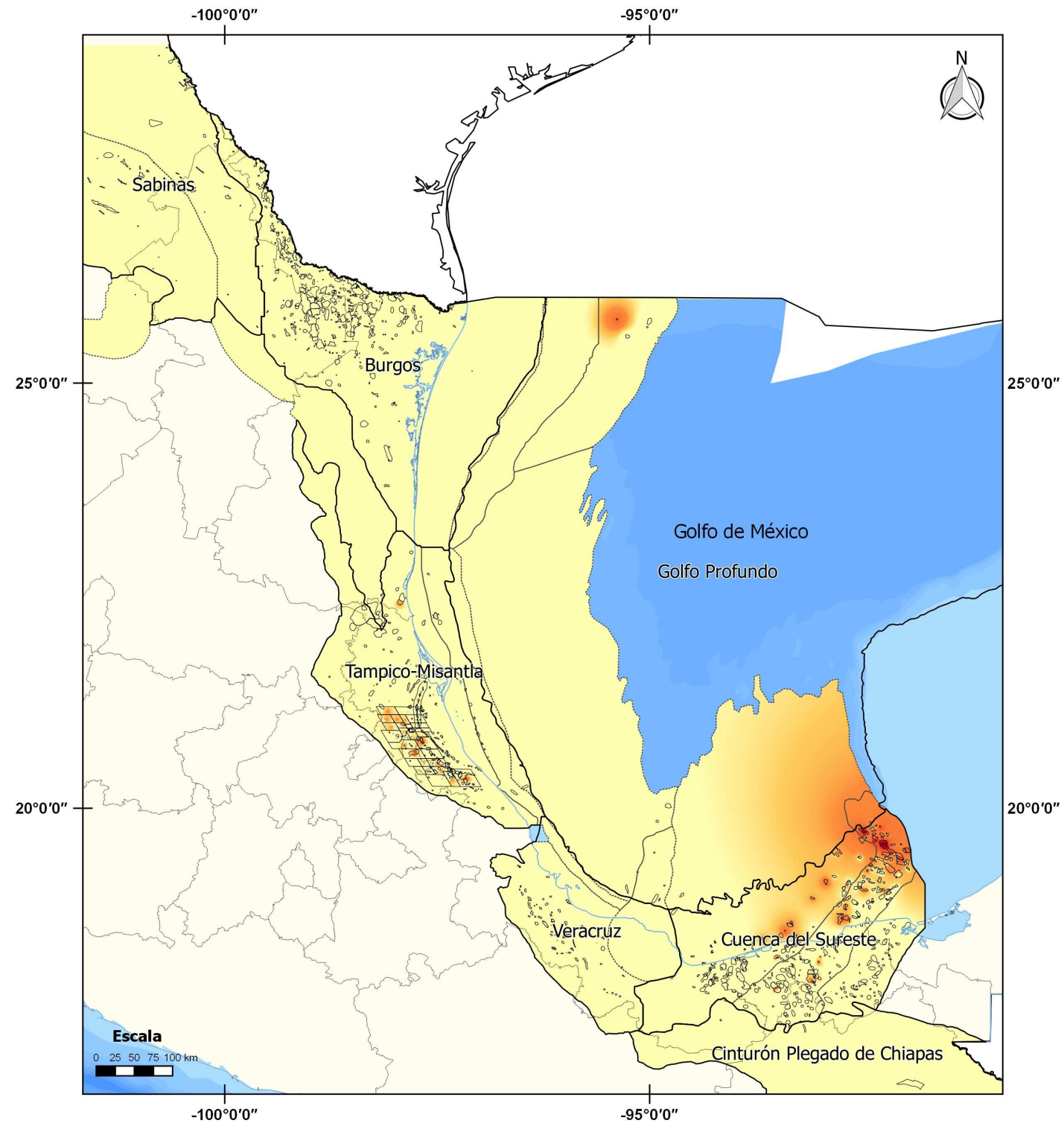

\begin{tabular}{|c|c|c|c|}
\hline \multicolumn{4}{|c|}{ Distribución de Reservas 3P de Crudo } \\
\hline 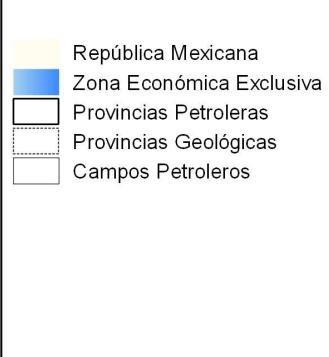 & 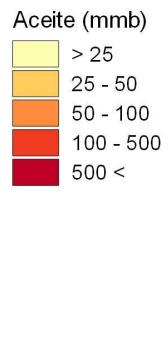 & $\begin{array}{l}\text { Clasificación } \\
\text { Pequeño } \\
\text { Mediano } \\
\text { Grande } \\
\text { Muy Grande } \\
\text { Gigante }\end{array}$ & $\begin{array}{c}\text { Reservas 3P de Crudo } \\
\text { Total Nacional } 19,455 \text { mmbpce } \\
\text { Total Cuencas del Sureste } 14,790 \text { mmbpce } \\
\text { Campo Mayor: Akal 2,602 mmbpce } \\
\text { Datos al } 1 \text { de Enero de } 2016 \\
\text { Clasificación de campos por su tamaño de } \\
\text { Ivanhoe y Luckie (1993). } \\
\text { Información de } \\
\text { Comisión Nacional de Hidrocarburos } \\
\text { Secretaría de Energía }\end{array}$ \\
\hline
\end{tabular}


Figura 9: Distribución del volumen original 1P de gas natural Figure 9: Distribution of the original 1P volume of natural gas

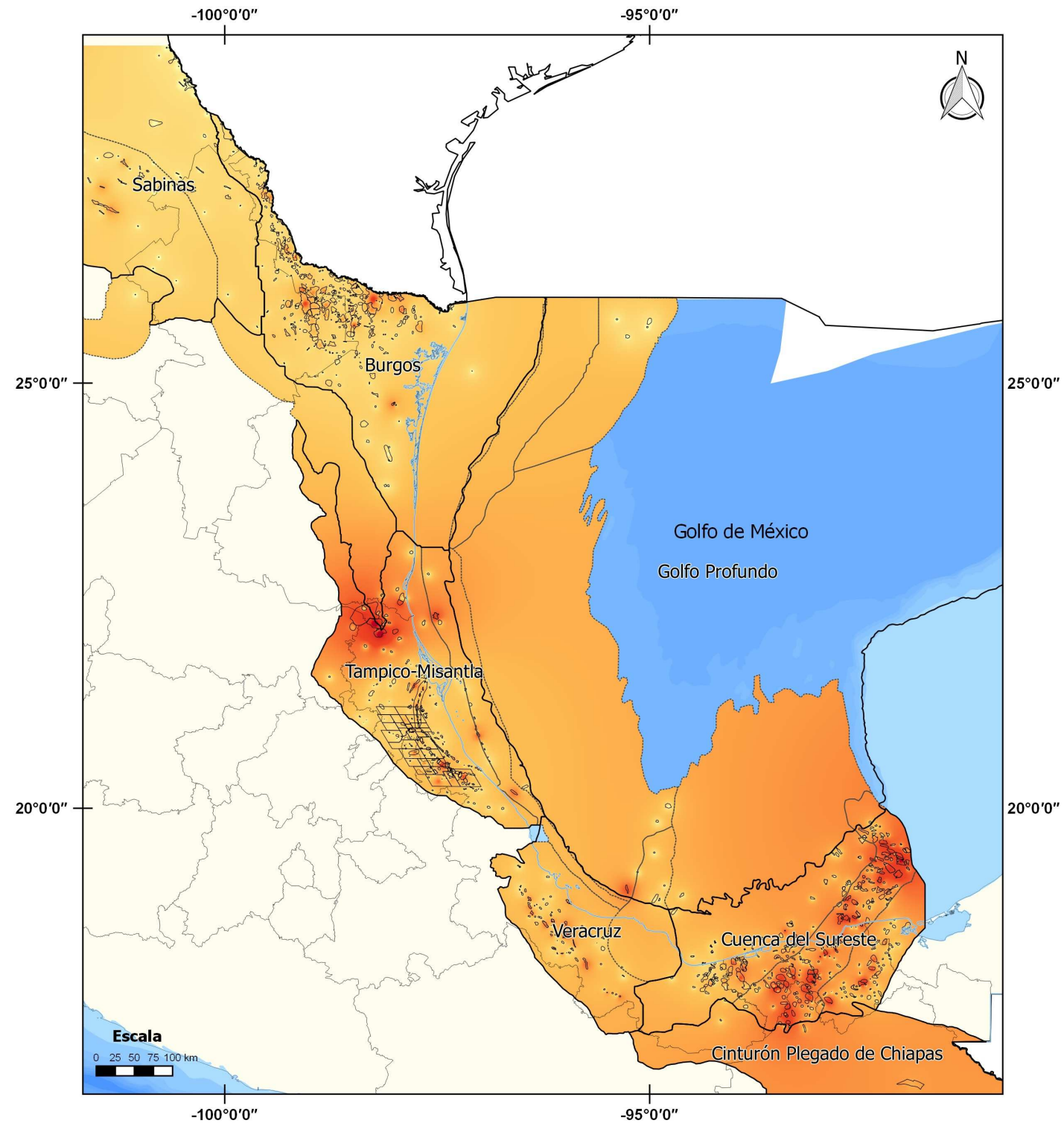

\begin{tabular}{|c|c|c|c|}
\hline \multicolumn{4}{|c|}{ Distribución de Volumen Original 1P de Gas Natural } \\
\hline $\begin{array}{l}\text { República Mexicana } \\
\text { Zona Económica Exclusiva } \\
\text { Provincias Petroleras } \\
\text { Provincias Geológicas } \\
\text { Campos Petroleros }\end{array}$ & $\begin{array}{l}\text { Gas (mmmpc) } \\
\begin{array}{|l|l} & 1 \\
\square & 1-10 \\
10-100 \\
100-250 \\
250-500 \\
500-1,000 \\
1,000-5,000 \\
5,000<\end{array}\end{array}$ & $\begin{array}{l}\text { Clasificación } \\
\text { Insignificante } \\
\text { Minúsculo } \\
\text { Muy Pequeño } \\
\text { Pequeño } \\
\text { Mediano } \\
\text { Grande } \\
\text { Muy Grande } \\
\text { Gigante }\end{array}$ & $\begin{array}{c}\text { Volumen Original 1P de Gas Natural } \\
\text { Total Nacional 198,566 mmmpc } \\
\text { Total Cuencas del Sureste } 122,359 \mathrm{mmmpc} \\
\text { Campo Mayor: Akal } 14,759 \mathrm{mmmpc} \\
\text { Datos al } 1 \text { de Enero de } 2016 \\
\text { Clasificación de campos por su tamaño de } \\
\text { Ivanhoe y Luckie (1993). } \\
\text { Información de } \\
\text { Comisión Nacional de Hidrocarburos } \\
\text { Secretaría de Energía }\end{array}$ \\
\hline
\end{tabular}


Figura 10: Distribución del volumen original 2P de gas natural

Figure 10: Distribution of the original 2P volume of natural gas

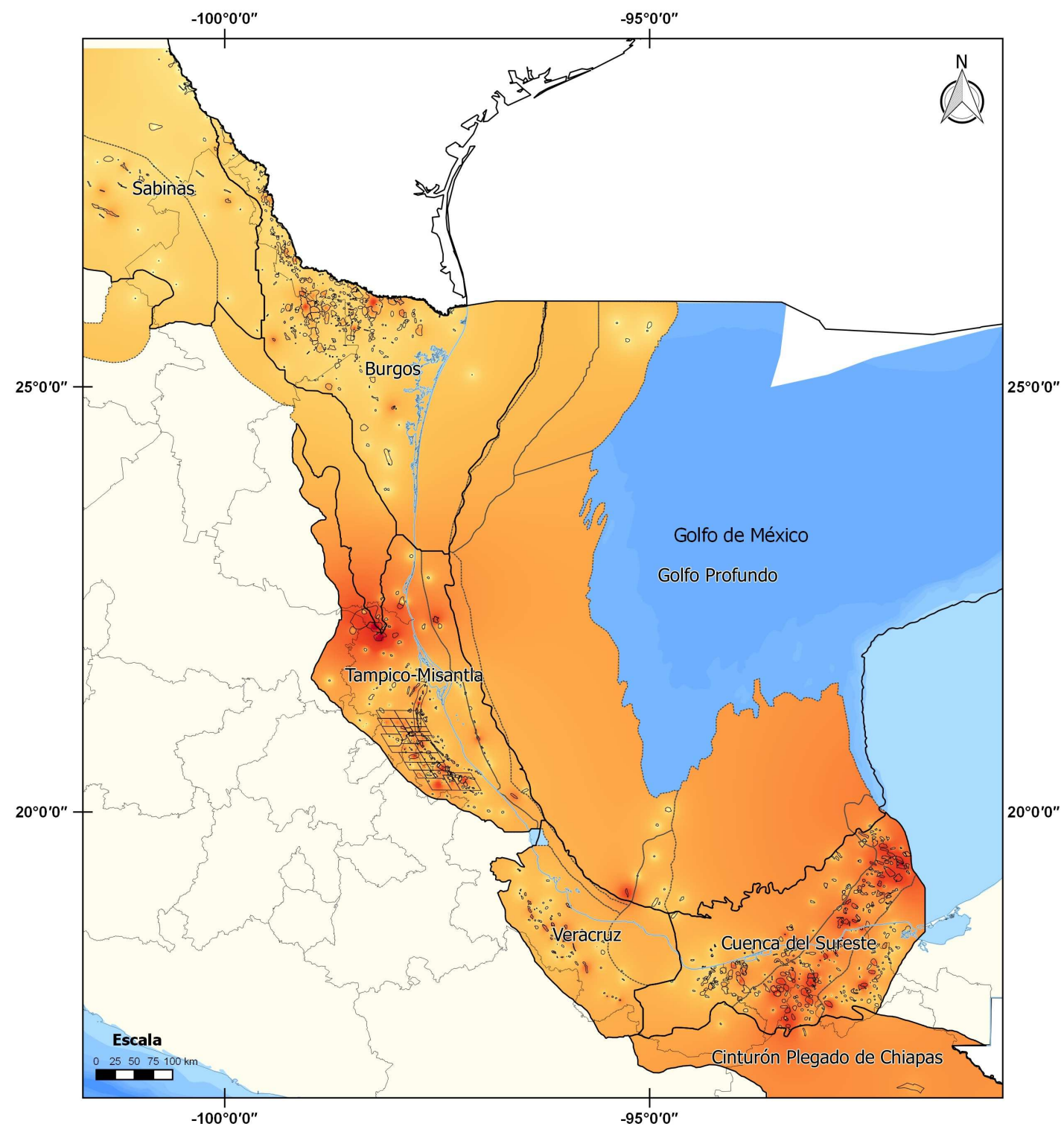

\begin{tabular}{|c|c|c|c|}
\hline \multicolumn{4}{|c|}{ Distribución de Volumen Original 2P de Gas Natural } \\
\hline $\begin{array}{l}\text { República Mexicana } \\
\text { Zona Económica Exclusiva } \\
\text { Provincias Petroleras } \\
\text { Provincias Geológicas } \\
\text { Campos Petroleros }\end{array}$ & $\begin{array}{l}\text { Gas (mmmpc) } \\
\begin{array}{|l|l} & 1 \\
\square & 1-10 \\
10-100 \\
100-250 \\
250-500 \\
500-1,000 \\
1,000-5,000 \\
5,000<\end{array}\end{array}$ & $\begin{array}{l}\text { Clasificación } \\
\text { Insignificante } \\
\text { Minúsculo } \\
\text { Muy Pequeño } \\
\text { Pequeño } \\
\text { Mediano } \\
\text { Grande } \\
\text { Muy Grande } \\
\text { Gigante }\end{array}$ & $\begin{array}{c}\text { Volumen Original 2P de Gas Natural } \\
\text { Total Nacional } 225,053 \mathrm{mmmpc} \\
\text { Total Cuencas del Sureste } 129,615 \mathrm{mmmpc} \\
\text { Campo Mayor: Akal } 14,791 \mathrm{mmmpc} \\
\text { Datos al } 1 \text { de Enero de } 2016 \\
\text { Clasificación de campos por su tamaño de } \\
\text { Ivanhoe y Luckie (1993). } \\
\text { Información de } \\
\text { Comisión Nacional de Hidrocarburos } \\
\text { Secretaría de Energía }\end{array}$ \\
\hline
\end{tabular}


Figura 11: Distribución del volumen original 3P de gas natural Figure 11: Distribution of the original 3P volume of natural gas

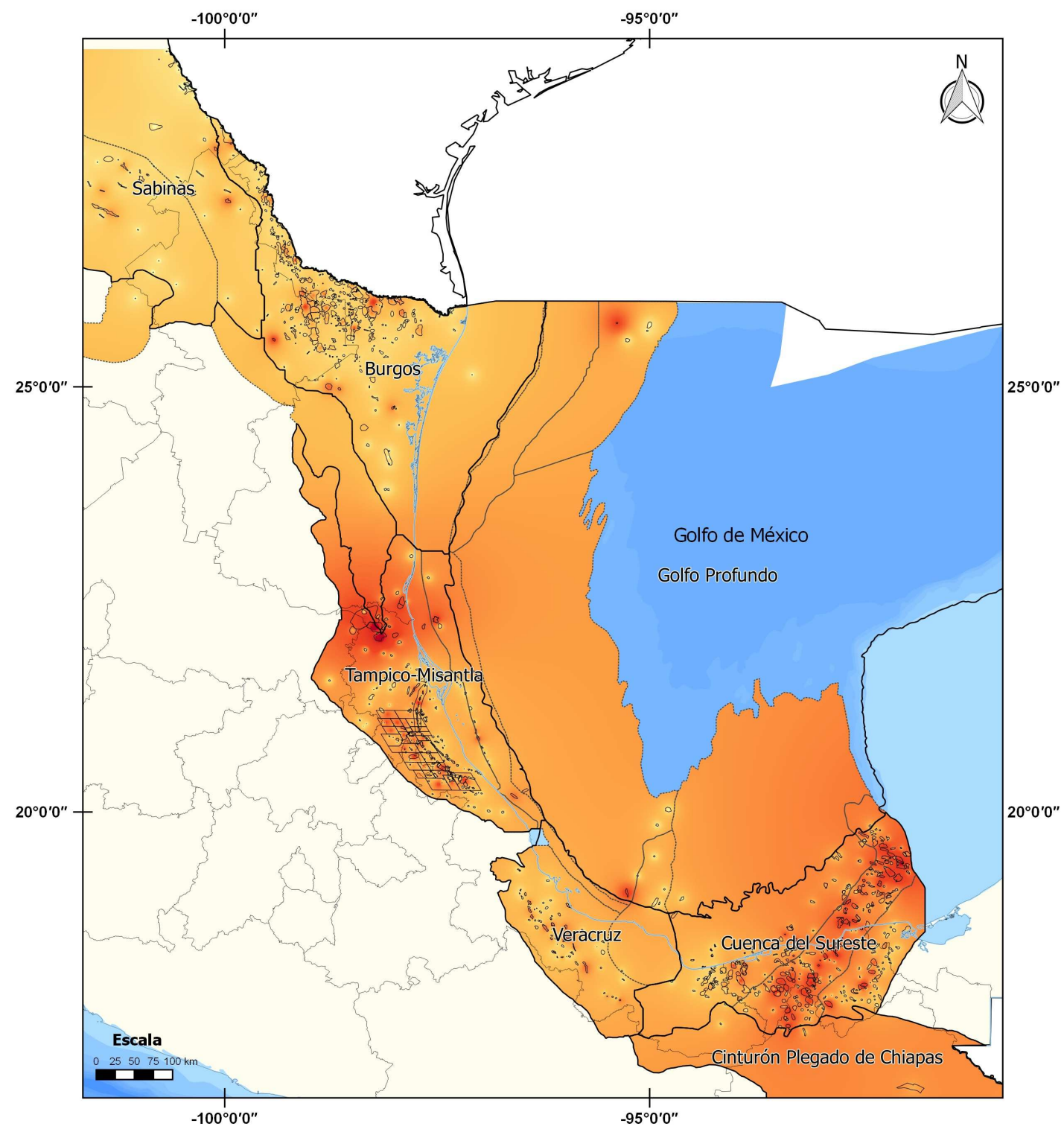

\begin{tabular}{|c|c|c|c|}
\hline \multicolumn{4}{|c|}{ Distribución de Volumen Original 3P de Gas Natural } \\
\hline $\begin{array}{l}\text { República Mexicana } \\
\text { Zona Económica Exclusiva } \\
\text { Provincias Petroleras } \\
\text { Provincias Geológicas } \\
\text { Campos Petroleros }\end{array}$ & $\begin{array}{l}\text { Gas (mmmpc) } \\
\begin{array}{|l|l} & 1 \\
\square & 1-10 \\
10-100 \\
100-250 \\
250-500 \\
500-1,000 \\
1,000-5,000 \\
5,000<\end{array}\end{array}$ & $\begin{array}{l}\text { Clasificación } \\
\text { Insignificante } \\
\text { Minúsculo } \\
\text { Muy Pequeño } \\
\text { Pequeño } \\
\text { Mediano } \\
\text { Grande } \\
\text { Muy Grande } \\
\text { Gigante }\end{array}$ & $\begin{array}{c}\text { Volumen Original 3P de Gas Natural } \\
\text { Total Nacional } 257,867 \mathrm{mmmpc} \\
\text { Total Cuencas del Sureste } 140,421 \mathrm{mmmpc} \\
\text { Campo Mayor: Pánuco } 15,135 \mathrm{mmmpc} \\
\text { Datos al } 1 \text { de Enero de } 2016 \\
\text { Clasificación de campos por su tamaño de } \\
\text { Ivanhoe y Luckie (1993). } \\
\text { Información de } \\
\text { Comisión Nacional de Hidrocarburos } \\
\text { Secretaría de Energía }\end{array}$ \\
\hline
\end{tabular}


Figura 12: Distribución de la Producción Acumulada de Gas Natural Figure 12: Distribution of cumulative natural gas production

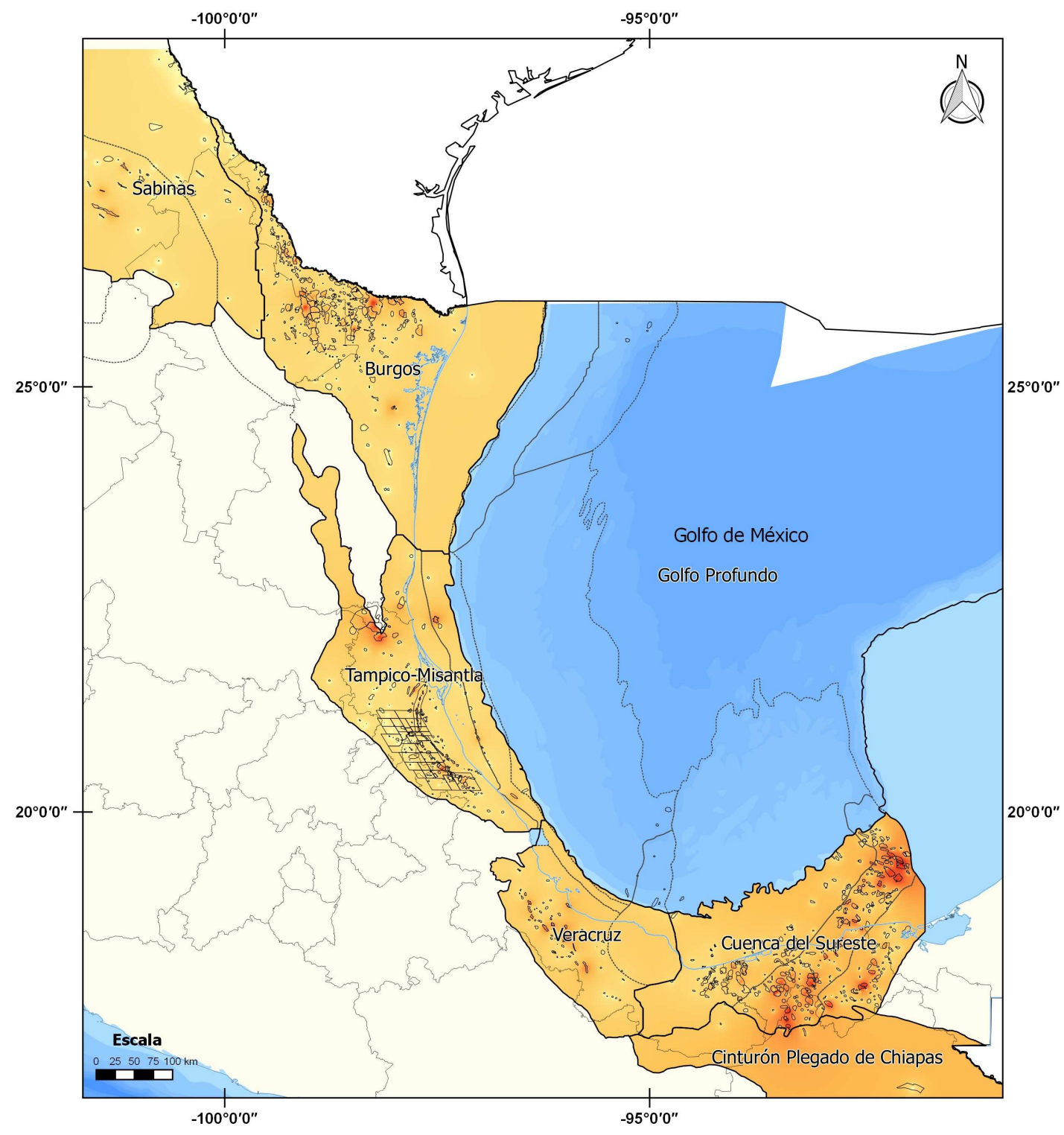

\begin{tabular}{|c|c|c|c|}
\hline \multirow[b]{2}{*}{$\begin{array}{l}\text { República Mexicana } \\
\text { Zona Económica Exclusiva } \\
\text { Provincias Petroleras } \\
\text { Provincias Geológicas } \\
\text { Campos Petroleros }\end{array}$} & \multicolumn{3}{|c|}{$\begin{array}{c}\text { Distribución de la Producción Acumulada } \\
\text { de Gas Natural }\end{array}$} \\
\hline & $\begin{array}{l}\text { Gas (mmmpc) } \\
\begin{array}{|l|l} & 1 \\
\square & 1-10 \\
10-100 \\
100-250 \\
& 250-500 \\
500-1,000 \\
1,000-5,000 \\
5,000<\end{array}\end{array}$ & $\begin{array}{l}\text { Clasificación } \\
\text { Insignificante } \\
\text { Minúsculo } \\
\text { Muy Pequeño } \\
\text { Pequeño } \\
\text { Mediano } \\
\text { Grande } \\
\text { Muy Grande } \\
\text { Gigante }\end{array}$ & $\begin{array}{c}\text { Producción Acumulada de Gas Natural } \\
\text { Total Nacional } 78,638 \mathrm{mmmpc} \\
\text { Total Cuencas del Sureste } 52,550 \mathrm{mmmpc} \\
\text { Campo Mayor: Akal } 8,256 \mathrm{mmmpc} \\
\text { Datos al 1 de Enero de } 2016 \\
\text { Clasificación de campos por su tamaño de } \\
\text { Ivanhoe y Luckie (1993). } \\
\text { Información de } \\
\text { Comisión Nacional de Hidrocarburos } \\
\text { Secretaría de Energía }\end{array}$ \\
\hline
\end{tabular}


Figura 13: Distribución de reservas 1P de Gas Natural

Figure 13: Distribution of 1P reserve of natural gas

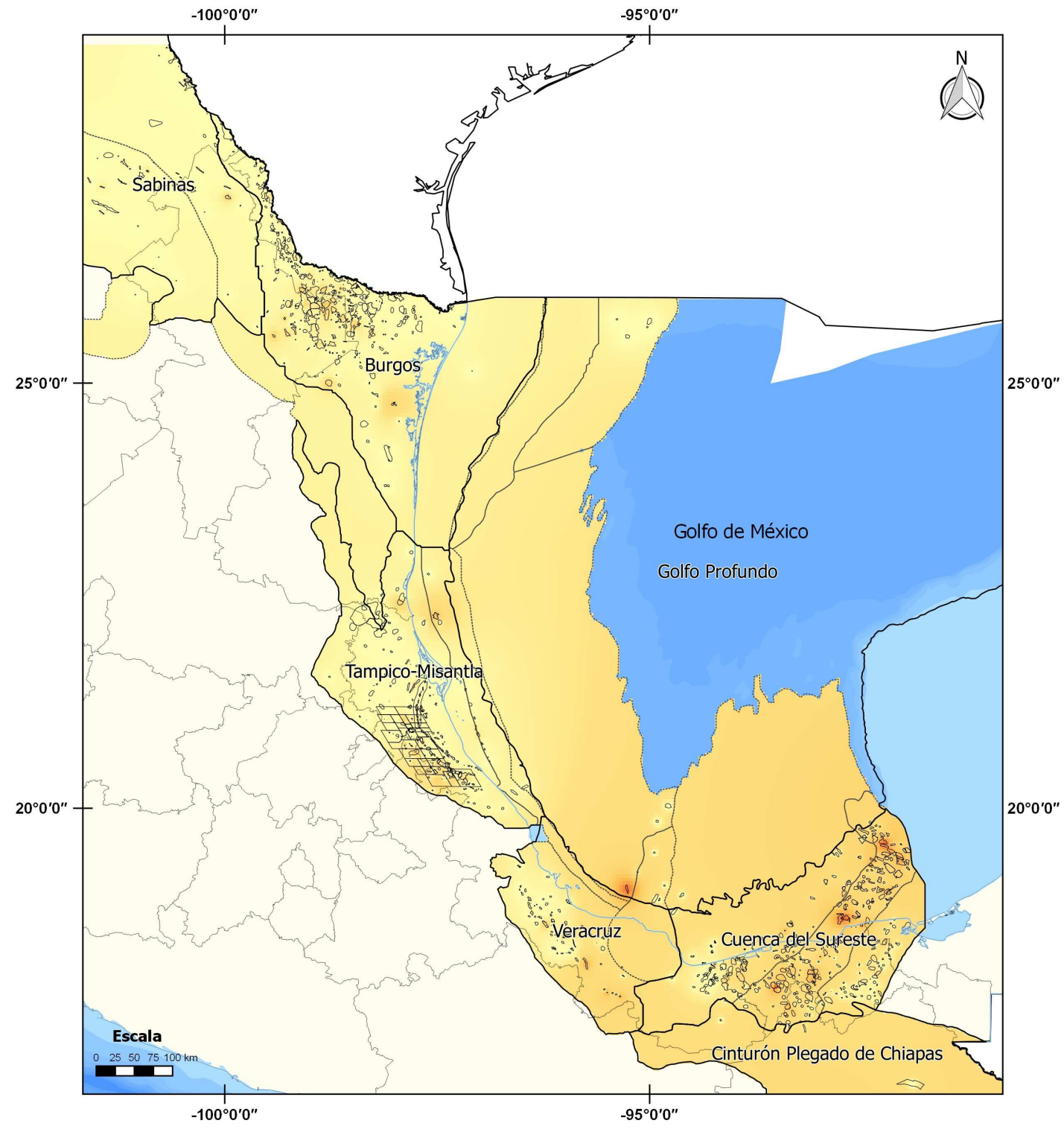

\begin{tabular}{|c|c|c|c|}
\hline \multirow[b]{2}{*}{$\begin{array}{l}\text { República Mexicana } \\
\text { Zona Económica Exclusiva } \\
\text { Provincias Petroleras } \\
\text { Provincias Geológicas } \\
\text { Campos Petroleros }\end{array}$} & \multicolumn{3}{|c|}{$\begin{array}{c}\text { Distribución de Reservas 1P de } \\
\text { Gas Natural }\end{array}$} \\
\hline & $\begin{array}{l}\text { Gas (mmmpc) } \\
\begin{array}{|l|l} & 1 \\
\square & 1-10 \\
10-100 \\
100-250 \\
& 250-500 \\
500-1,000 \\
1,000-5,000 \\
5,000< \\
\square\end{array}\end{array}$ & $\begin{array}{l}\text { Clasificación } \\
\text { Insignificante } \\
\text { Minúsculo } \\
\text { Muy Pequeño } \\
\text { Pequeño } \\
\text { Mediano } \\
\text { Grande } \\
\text { Muy Grande } \\
\text { Gigante }\end{array}$ & $\begin{array}{c}\text { Clasificación de campos por su tamaño de } \\
\text { Ivanhoe y Luckie (1993). } \\
\text { Información de } \\
\text { Comisión Nacional de Hidrocarburos } \\
\text { Secretaría de Energía }\end{array}$ \\
\hline
\end{tabular}


Figura 14: Distribución de reservas 2P de Gas Natural

Figure 14: Distribution of $2 P$ reserve of natural gas

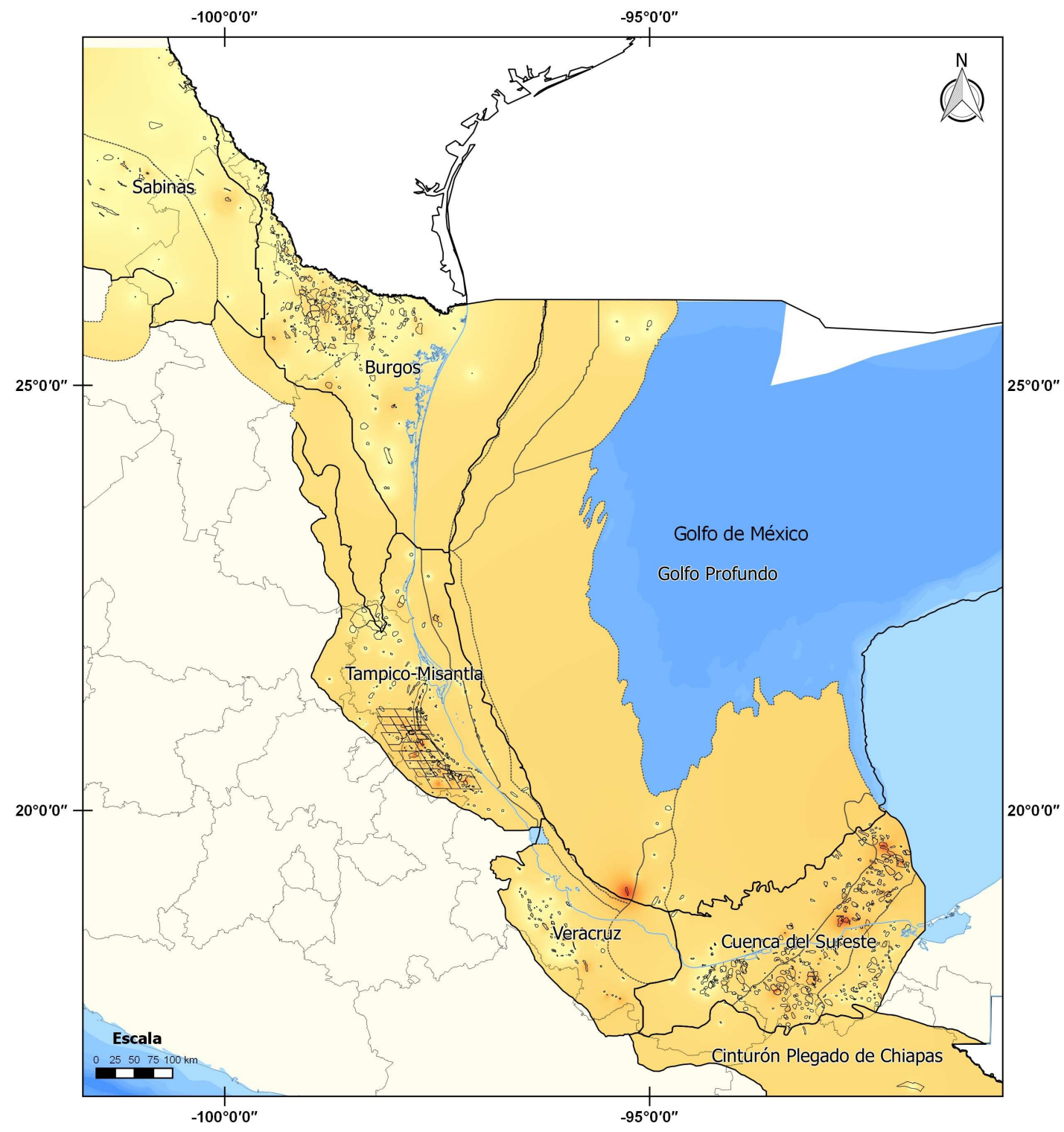

\begin{tabular}{|c|c|c|c|}
\hline \multirow[b]{2}{*}{$\begin{array}{l}\text { República Mexicana } \\
\text { Zona Económica Exclusiva } \\
\text { Provincias Petroleras } \\
\text { Provincias Geológicas } \\
\text { Campos Petroleros }\end{array}$} & \multicolumn{3}{|c|}{$\begin{array}{c}\text { Distribución de Reservas 2P de } \\
\text { Gas Natural }\end{array}$} \\
\hline & $\begin{array}{l}\text { Gas (mmmpc) } \\
\begin{array}{|l|l} & 1 \\
\square & 1-10 \\
10-100 \\
100-250 \\
& 250-500 \\
500-1,000 \\
1,000-5,000 \\
5,000< \\
\square\end{array}\end{array}$ & $\begin{array}{l}\text { Clasificación } \\
\text { Insignificante } \\
\text { Minúsculo } \\
\text { Muy Pequeño } \\
\text { Pequeño } \\
\text { Mediano } \\
\text { Grande } \\
\text { Muy Grande } \\
\text { Gigante }\end{array}$ & $\begin{array}{c}\text { Clasificación de campos por su tamaño de } \\
\text { Ivanhoe y Luckie (1993). } \\
\text { Información de } \\
\text { Comisión Nacional de Hidrocarburos } \\
\text { Secretaría de Energía }\end{array}$ \\
\hline
\end{tabular}


Figura 15: Distribución de reservas 3P de Gas Natural

Figure 15: Distribution of 3P reserve of natural gas

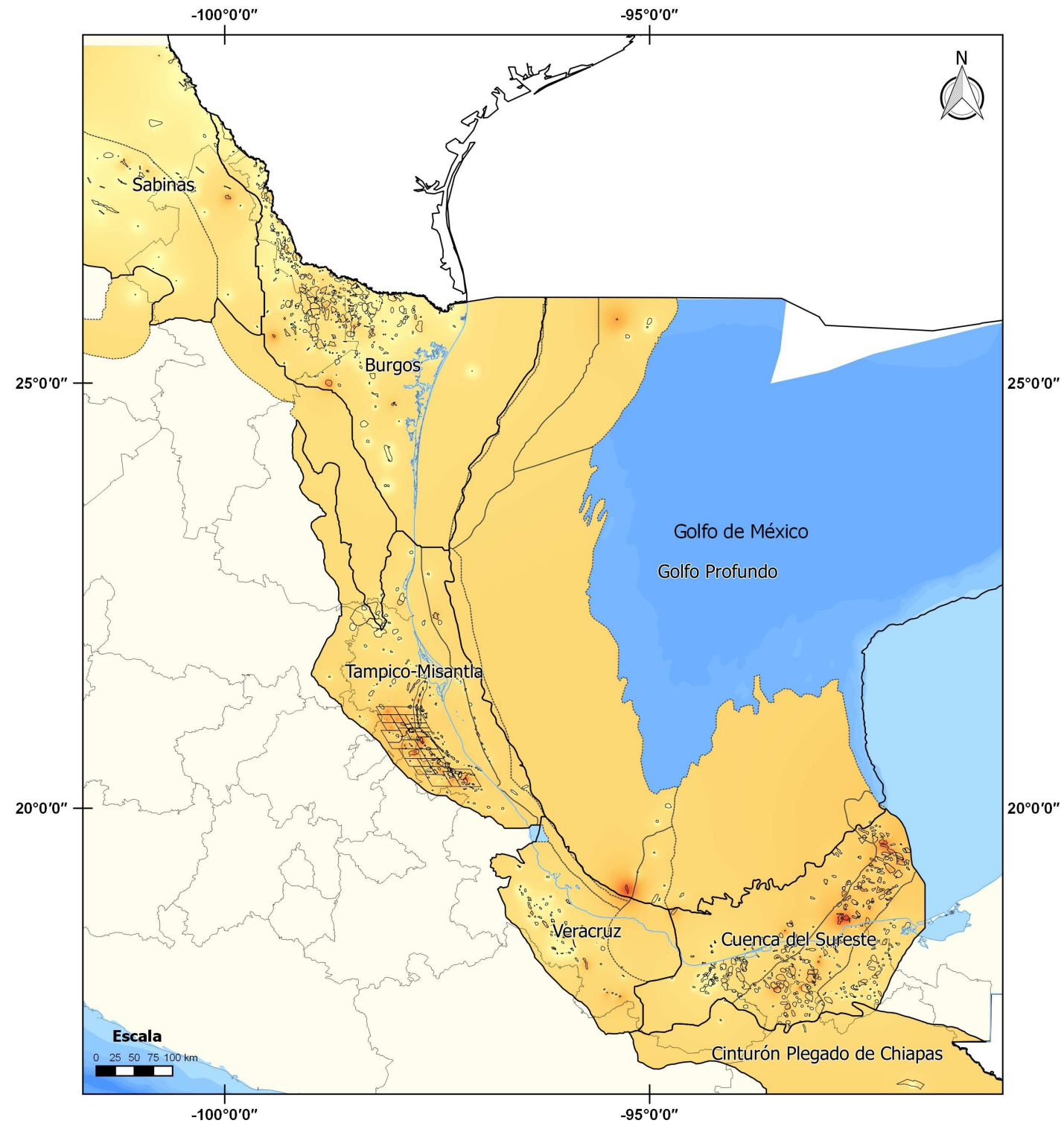

\begin{tabular}{|c|c|c|c|}
\hline \multirow[b]{2}{*}{$\begin{array}{l}\text { República Mexicana } \\
\text { Zona Económica Exclusiva } \\
\text { Provincias Petroleras } \\
\text { Provincias Geológicas } \\
\text { Campos Petroleros }\end{array}$} & \multicolumn{3}{|c|}{$\begin{array}{c}\text { Distribución de Reservas 3P de } \\
\text { Gas Natural }\end{array}$} \\
\hline & $\begin{array}{l}\text { Gas (mmmpc) } \\
\begin{array}{|l|l} & 1 \\
\square & 1-10 \\
10-100 \\
100-250 \\
& 250-500 \\
500-1,000 \\
1,000-5,000 \\
5,000<\end{array}\end{array}$ & $\begin{array}{l}\text { Clasificación } \\
\text { Insignificante } \\
\text { Minúsculo } \\
\text { Muy Pequeño } \\
\text { Pequeño } \\
\text { Mediano } \\
\text { Grande } \\
\text { Muy Grande } \\
\text { Gigante }\end{array}$ & $\begin{array}{c}\text { Reservas 3P de Gas Natural } \\
\text { Total Nacional } 32,568 \mathrm{mmmpc} \\
\text { Total Cuencas del Sureste 16,187 mmmpc } \\
\text { Campo Mayor: Tsimín 1,397 mmmpc } \\
\text { Datos al } 1 \text { de Enero de } 2016 \\
\text { Clasificación de campos por su tamaño de } \\
\text { Ivanhoe y Luckie (1993). } \\
\text { Información de } \\
\text { Comisión Nacional de Hidrocarburos } \\
\text { Secretaría de Energía }\end{array}$ \\
\hline
\end{tabular}


Figura 16: Distribución de reservas 1P de Petróleo Crudo Equivalente Figure 16: Distribution of total hydrocarbon 1P reserves (in barrel of oil equivalent)

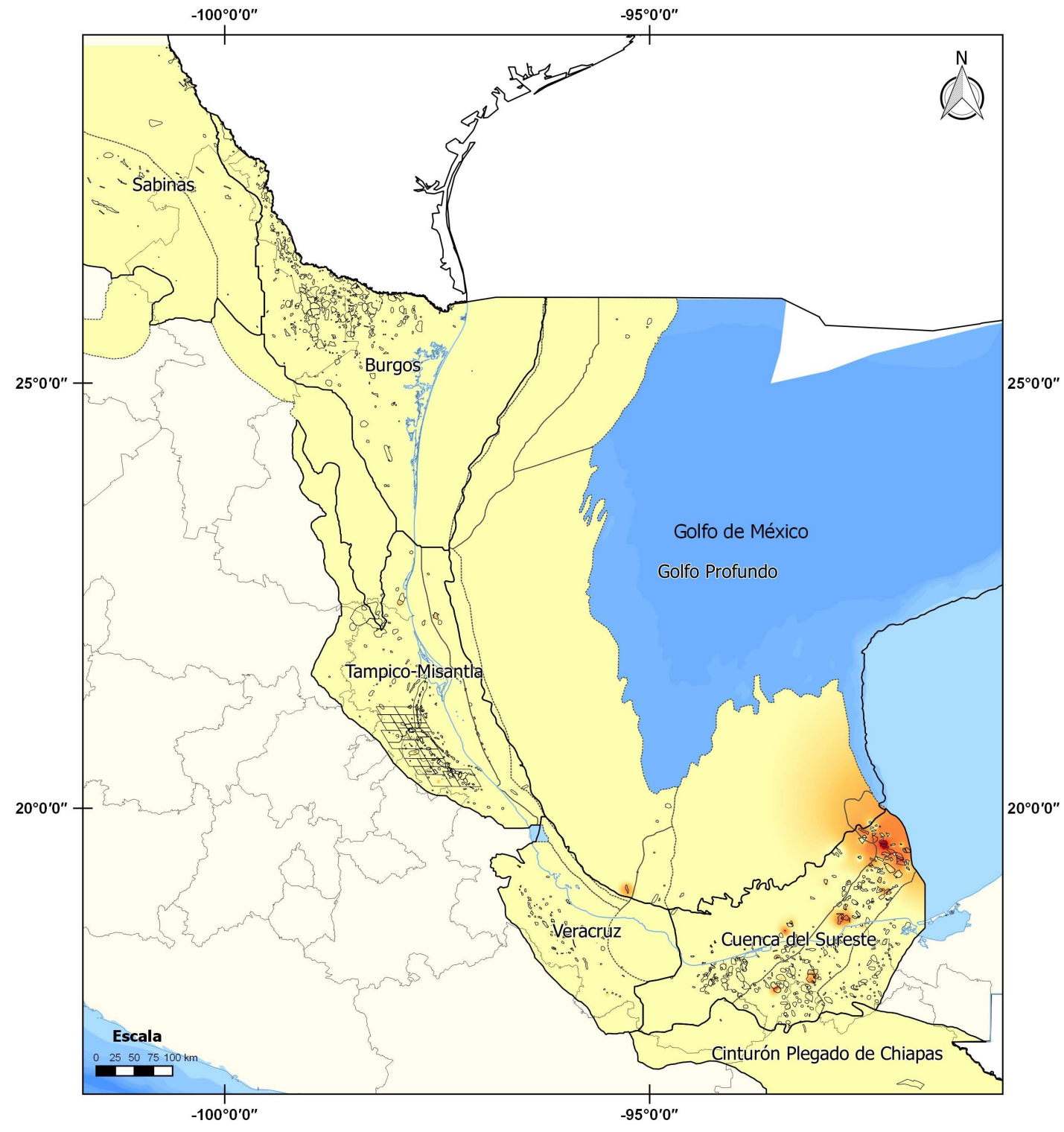

Distribución de Reservas 1P de Petróleo Crudo Equivalente

República Mexicana

Zona Económica Exclusiva

Provincias Petroleras

Provincias Geológicas

Campos Petroleros

\begin{tabular}{|c|c|}
\hline PCE (mmbpce) & Clasificación \\
\hline$>25$ & Pequeño \\
\hline $25-50$ & Mediano \\
\hline $50-100$ & Grande \\
\hline $100-500$ & Muy Grande \\
\hline $500<$ & Gigante \\
\hline
\end{tabular}

Reservas $1 \mathrm{P}$ de Petróleo Crudo Equivalente Total Nacional 10,243 mmbpce Total Cuencas del Sureste $8,855 \mathrm{mmbpce}$ Campo Mayor: Maloob 1,657 mmbpce Datos al 1 de Enero de 2016

Clasificación de campos por su tamaño de Ivanhoe y Luckie (1993).

Información de Comisión Nacional de Hidrocarburos Secretaría de Energía 
Figura 17: Distribución de reservas 2P de Petróleo Crudo Equivalente Figure 17: Distribution of total hydrocarbon 2P reserves (in barrel of oil equivalent)

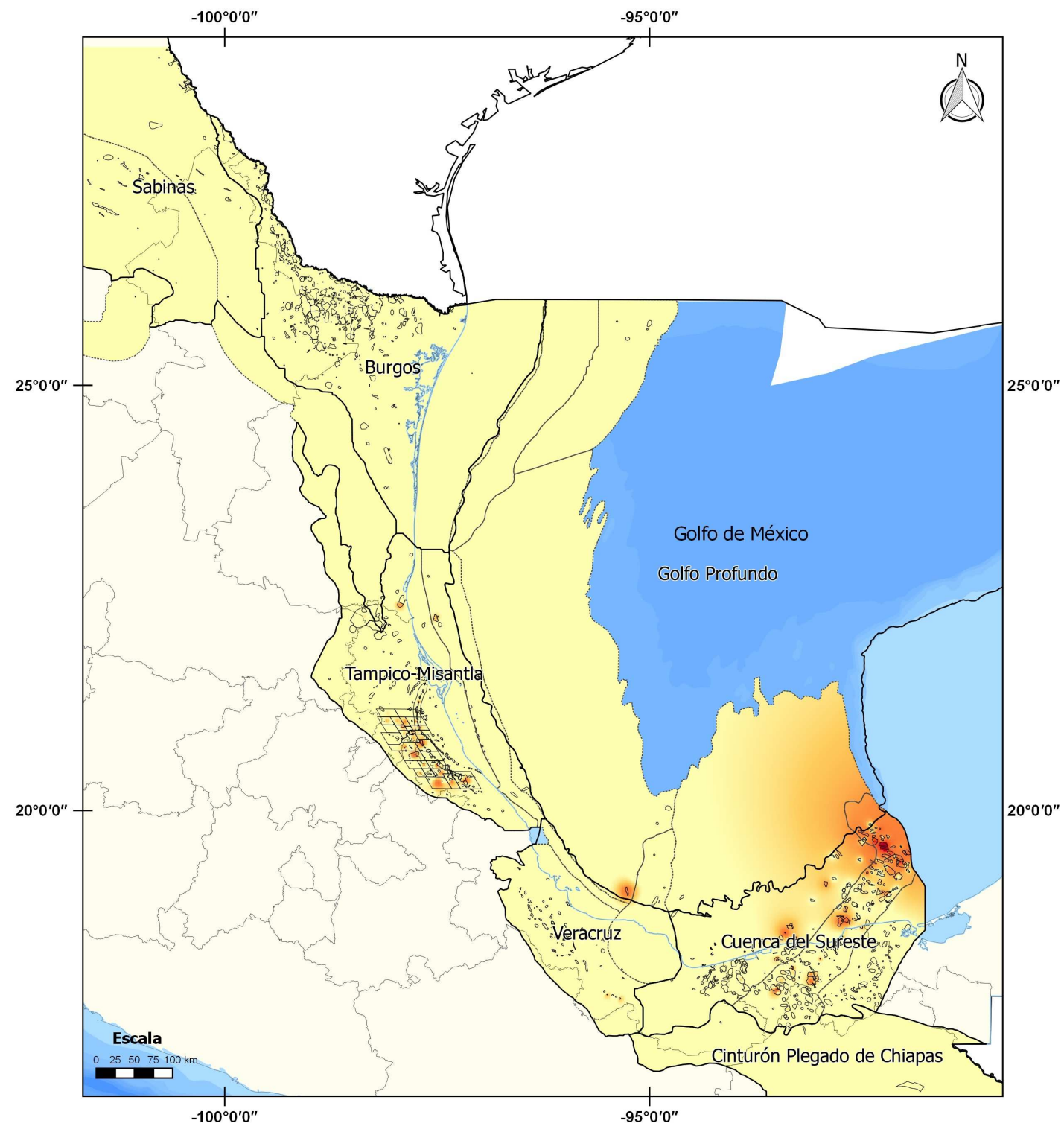

Distribución de Reservas 2P de Petróleo Crudo Equivalente

República Mexicana

Zona Económica Exclusiva

Provincias Petroleras

Provincias Geológicas

Campos Petroleros
PCE (mmbpce) Clasificación

$\begin{array}{ll}>25 & \text { Pequeño } \\ 25-50 & \text { Mediano } \\ 50-100 & \text { Grande } \\ 100-500 & \text { Muy Grande } \\ 500< & \text { Gigante }\end{array}$

Reservas $2 \mathrm{P}$ de Petróleo Crudo Equivalente Total Nacional 17,792 mmbpce Total Cuencas del Sureste 13,038 mmbpce Campo Mayor: Akal 1,918 mmbpce Datos al 1 de Enero de 2016

Clasificación de campos por su tamaño de Ivanhoe y Luckie (1993).

Información de Comisión Nacional de Hidrocarburos Secretaría de Energía 
Figura 18: Distribución de reservas 3P de Petróleo Crudo Equivalente Figure 18: Distribution of total hydrocarbon 3P reserves (in barrel of oil equivalent)

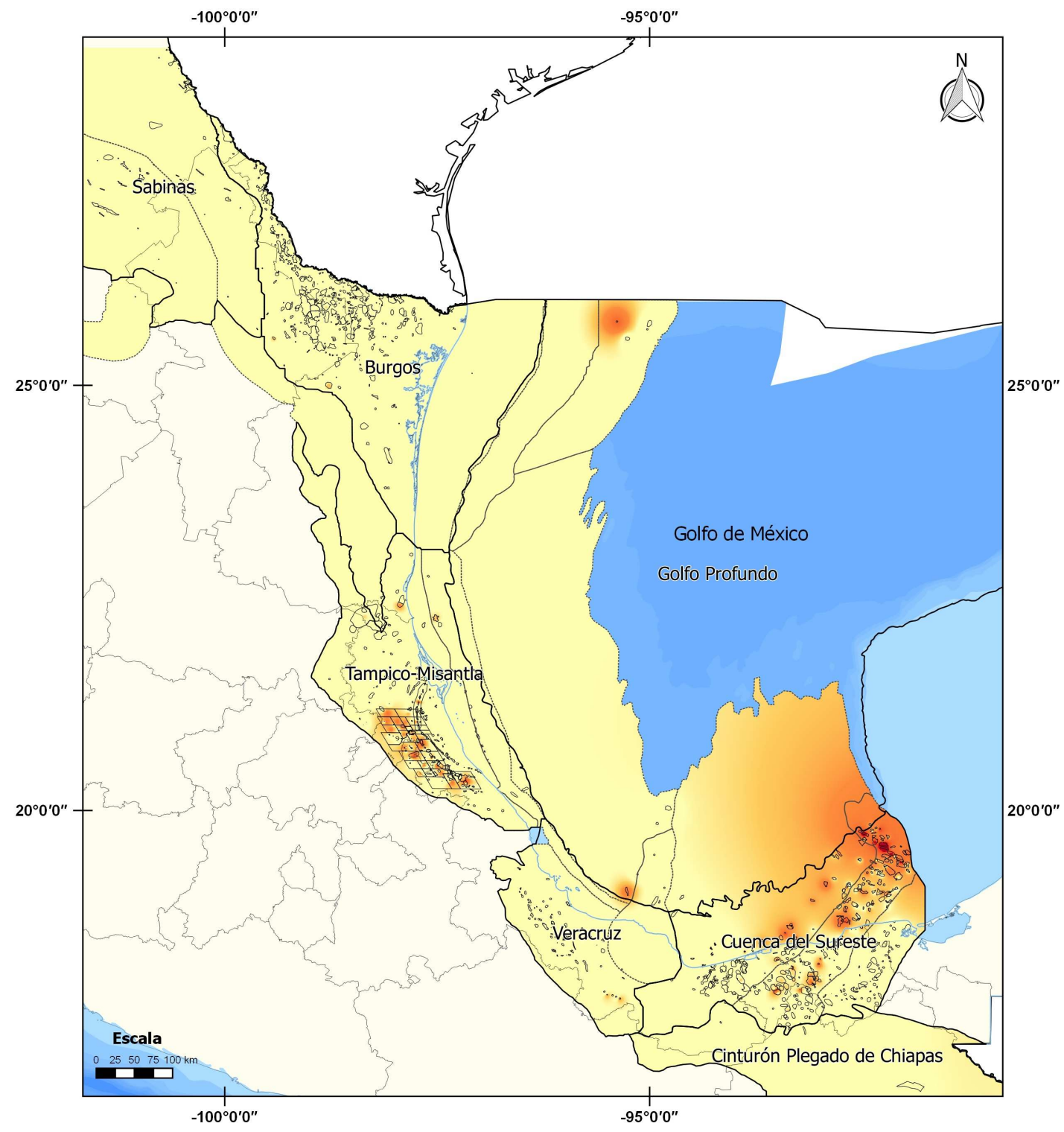

Distribución de Reservas 3P de Petróleo Crudo Equivalente

República Mexicana

Zona Económica Exclusiva

Provincias Petroleras

Provincias Geológicas

Campos Petroleros

\begin{tabular}{|c|c|}
\hline PCE (mmbpce) & Clasificación \\
\hline$>25$ & Pequeño \\
\hline $25-50$ & Mediano \\
\hline $50-100$ & Grande \\
\hline $100-500$ & Muy Grande \\
\hline $500<$ & Gigante \\
\hline
\end{tabular}

Reservas $3 \mathrm{P}$ de Petróleo Crudo Equivalente Total Nacional 26,140 mmbpce Total Cuencas del Sureste 18,095 mmbpce Campo Mayor: Akal 2,834 mmbpce Datos al 1 de Enero de 2016

Clasificación de campos por su tamaño de Ivanhoe y Luckie (1993).

Información de Comisión Nacional de Hidrocarburos Secretaría de Energía 


\section{Figura 19: Distribución de la Producción Acumulada de Petróleo Crudo}

Equivalente

Figure 19: Distribution of cumulative total hydrocarbon (in barrel of oil equivalent) production

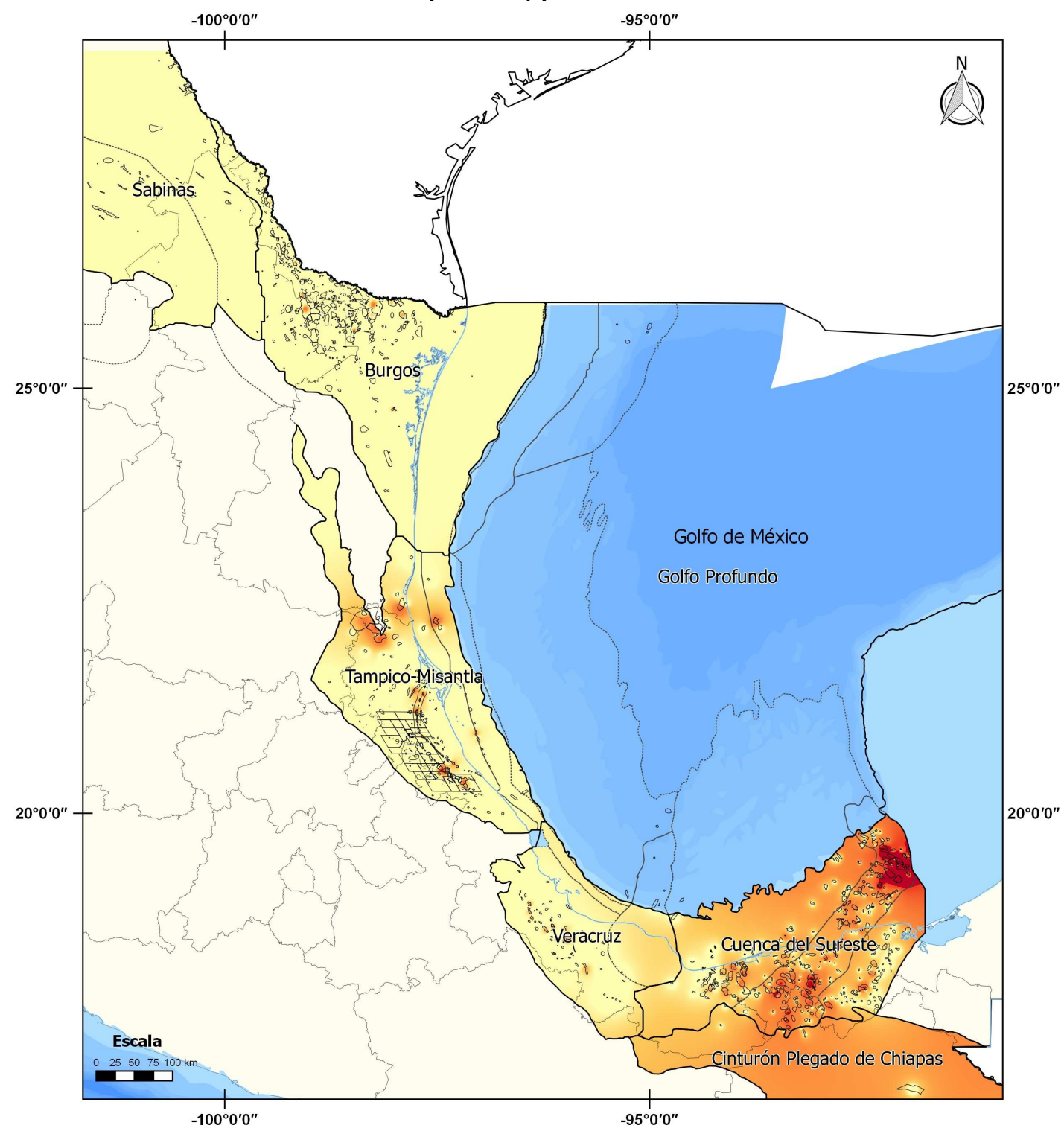

\begin{tabular}{|c|c|c|c|}
\hline \multirow{7}{*}{$\begin{array}{l}\text { República Mexicana } \\
\text { Zona Económica Exclusiva } \\
\text { Provincias Petroleras } \\
\text { Provincias Geológicas } \\
\text { Campos Petroleros }\end{array}$} & \multicolumn{2}{|c|}{$\begin{array}{c}\text { Distribución de la Producción Acumulada } \\
\text { Petróleo Crudo Equivalente }\end{array}$} & \multirow{8}{*}{$\begin{array}{l}\text { nulada } \\
\text { Producción Acumulada de Petróleo Crudo } \\
\text { Equivalente } \\
\text { Total Nacional } 57,788 \text { mmbpce } \\
\text { Total Cuencas del Sureste } 48,360 \text { mmbpce } \\
\text { Campo Mayor: Akal } 14,403 \mathrm{mmbpce} \\
\text { Datos al } 1 \text { de Enero de } 2016 \\
\text { Clasificación de campos por su tamaño de } \\
\text { Ivanhoe y Luckie (1993). }\end{array}$} \\
\hline & PCE (mmbpce) & Clasificación & \\
\hline & $>25$ & Pequeño & \\
\hline & $25-50$ & Mediano & \\
\hline & $50-100$ & Grande & \\
\hline & $100-500$ & Muy Grande & \\
\hline & $500<$ & Gigante & \\
\hline & & & \\
\hline & & & $\begin{array}{l}\text { Información de } \\
\text { Comisión Nacional de Hidrocarburos } \\
\text { Secretaría de Energía }\end{array}$ \\
\hline
\end{tabular}


En el caso del gas natural los yacimientos corresponden principalmente a gas asociado, lo cual es un reflejo de las condiciones de los yacimientos de crudo, por lo que la mayor parte del volumen original se concentra en la zona norte de la Provincia de Tampico Misantla y en la provincia de Pilar Reforma Akal de las Cuencas del Sureste (Figuras 9 a 11), siendo dichas áreas las principales productoras (Figura 12). Actualmente se encuentran en etapa de agotamiento, como lo muestran los mapas de la distribución de reservas 1P, 2P y 3P (Figuras 13 a 15).

La última serie de mapas visualiza las reservas conjuntas de hidrocarburos expresadas como petróleo crudo equivalente, es decir sumando aceite crudo, condensado, líquidos de plantas y gas seco equivalente a líquido (PEMEX, 2010). La distribución de las reservas 1P, 2P y 3P, así como la producción acumulada (Figuras 16 a 19) reflejan esencialmente la distribución de crudo ya que este representa el $75 \%$ de las reservas de hidrocarburos.

\section{Software}

Para elaboración y diseño de los mapas se utilizó el software libre QGIS 2.14.3-Essen. Las bases de datos de origen se elaboraron mediante la aplicación Microsoft Excel de la suite Microsoft Office 2013.

\section{Datos}

Con el Proyecto se generaron bases de datos y conjuntos de objetos en formato vectorial (polígonos y puntos) y ráster. La información está contenida en un sistema de información geográfica bajo el Sistema de Referencia de Coordenadas Geográficas, datum WGS 84. Las características de la información compilada y generada se encuentran en las Tablas 2 y 3 .

Tabla 2: Datos Generados por IDW

Table 2: Data generated by IDW

\begin{tabular}{ccc}
\hline $\begin{array}{c}\text { Recurso } \\
\text { Clasificación }\end{array}$ & Categoría & Tipo \\
\hline $\begin{array}{c}\text { Petróleo Crudo Equivalente } \\
\text { Reservas Remanentes }\end{array}$ & $1 \mathrm{P}$ & \\
& $2 \mathrm{P}$ & Ráster \\
Producción Acumulada & $3 \mathrm{P}$ & \\
\hline Crudo & & \\
Volumen Original - & $1 \mathrm{P}$ & \\
Reservas Remanentes & $2 \mathrm{P}$ & Ráster \\
Producción Acumulada & $3 \mathrm{P}$ & \\
Gas Natural & & \\
Volumen Original - & $1 \mathrm{P}$ & \\
Reservas Remanentes & $2 \mathrm{P}$ & Ráster \\
Producción Acumulada & $3 \mathrm{P}$ & \\
\hline
\end{tabular}

\section{Agradecimientos}

Este trabajo fue financiado por el Programa de Apoyo a Proyectos para la Innovación y Mejoramiento de la Enseñanza (Proyecto PE105415). DHM agradece al Consejo Nacional de Ciencia y Tecnología de México (Conacyt) por el apoyo con una beca de posgrado.

Tabla 3: Datos Compilados y sus características

Table 3: Compiled data and their characteristics

\begin{tabular}{clll}
\hline Atributo & $\begin{array}{l}\text { Número de } \\
\text { Elementos }\end{array}$ & Tipo & Origen \\
\hline Campos & 1,10 & Poligono & Compilación \\
\hline Provincia & 7 & Poligono & CNIH \\
Petrolera & & & \\
Provincia & 16 & Poligono & \\
Geológica & & & \\
1P RR PCE & 761 & Numérico & \\
2P RR PCE & 761 & Numérico & \\
3P RR PCE & 761 & Numérico & \\
PA PCE & 761 & Numérico & \\
1P RR Crudo & 761 & Numérico & \\
2P RR Crudo & 761 & Numérico & \\
3P RR Crudo & 761 & Numérico & \\
1P VO Crudo & 761 & Numérico & \\
2P VO Crudo & 761 & Numérico & CNH \\
3P VO Crudo & 761 & Numérico & \\
PA Crudo & 761 & Numérico & \\
1P RR Gas & 761 & Numérico & \\
2P RR Gas & 761 & Numérico & \\
3P RR Gas & 761 & Numérico & \\
1P VO Gas & 761 & Numérico & \\
2P VO Gas & 761 & Numérico & \\
3P VO Gas & 761 & Numérico & \\
PA Gas & 761 & Numérico & \\
\hline
\end{tabular}

\section{Referencias}

Alvarez de la Borda, J., 2006. Crónica del petróleo en México: De 1863 hasta nuestros días. (1st ed.). México, D.F: Petróleos Mexicanos, 171 pp.

Banco Mundial, 2016. Rentas del petróleo como \% del PIB. http:// datos . bancomundial .org/indicador/NY. GDP. PETR.RT . ZS, Consultado: Agosto 2017.

Barker, G.J., 2008. Application of the PRMS to coal seam gas. Society of Petroleum Engineers Asia Pacific Oil \& Gas Conference and Exhibition, SPE Paper 117124, Perth, Australia, 13 pp.

Bentham, J., 2014. The scenario approach to possible futures for oil and natural gas. Energy Policy 64 (Supplement C), 87-92, doi: 10.1016/j.enpol.2013.08.019, http://www.sciencedirect.com/ science/article/pii/S0301421513008124.

British Petroleum, 2016. BP Statistical Review of World Energy. London: British Petroleum Co.

Burrough, P. A., McDonnell, 1998. Principles of Geographical Information Systems. Oxford University Press, 333 pp.

Comisión Nacional de Hidrocarburos (CNH), 2012. Análisis de Información de las Reservas de Hidrocarburos de México al 1 de enero del 2012, 7 p.

Comisión Nacional de Hidrocarburos (CNH), 2016. Centro Nacional de Información de Hidrocarburos (en línea). http://portal.cnih.cnh.gob. $\mathrm{mx} / \mathrm{iicnih} /$ ?lng=es_MX, Consultado abril 2016.

Comisión Nacional de Hidrocarburos (CNH), 2017a. Estadísticas, recursos y reservas, reservas por campo 2016. Centro Nacional de Información de Hidrocarburos. http: //portal. cnih.cnh.gob.mx/estadisticas . php, Consultado mayo 2017. 
Comisión Nacional de Hidrocarburos (CNH), 2017b. Resolución CNH.01.001/17 relativa al procedimiento de revisión de las estimaciones de Reservas 2P y 3P del Campo Akal, por la que la $\mathrm{CNH}$ consolida y publica valores de las reservas $2 \mathrm{P}$ y $3 \mathrm{P}$ de hidrocarburos de la Nación al 1 de enero de 2016.1a Sesión Ordinaria del Órgano de Gobierno. Ciudad de México. 27 de enero de 2017.

Diario Oficial de la Federación (DOF), 2014. Acuerdo por el que se establece el procedimiento para delimitar las áreas susceptibles de adjudicarse a través de asignaciones. México, 12 de agosto de 2014.

Hernández Martínez, D., 2017. La Producción Petrolera Mexicana: Análisis Histórico y Escenario a Futuro. Universidad Nacional Autónoma de México, Posgrado en Ciencias de la Tierra, Centro de Geociencias, Tesis de maestría, $152 \mathrm{pp}$.

Instituto Nacional de Estadística y Geografía (INEGI), 2015. Estadísticas históricas de México 2014. México, Cuadro 17.24.

Ivanhoe, L. F., Leckie, G., 1993. Global Oil, Gas Fields, Sizes Tallied, Analyzed. Oil and Gas Journal 91 (7), United States, https://www .osti.gov/ scitech/biblio/5940467.

Longley, P., 2005. Geographic Information Systems and Science. John Wiley $\&$ Sons.

Petróleos Mexicanos (PEMEX), 2010. Las Reservas de Hidrocarburos de Mé- xico. Petróleos Mexicanos.

Society of Petroleum Engineers (SPE), American Association of Petroleum Geologists (AAPG), World Petroleum Council (WPC), Society of Petroleum Evaluation Engineers (SPEE), 2007. Petroleum Resources Management System, 47 pp. http://www.spe.org/industry/docs/Petroleum_ Resources_Management_System_2007.pdf.

Society of Petroleum Engineers (SPE), American Association of Petroleum Geologists (AAPG), World Petroleum Council (WPC), Society of Petroleum Evaluation Engineers (SPEE), Society of Exploration Geophysicists (SEG), 2011. Guidelines for Application of the Petroleum Resources Management System, 222 pp. http://www.spe.org/industry/docs/PRMS_ Guidelines_Nov2011.pdf.

Xu, H., Wu, Q., Lei, H., Li, S., 2012. Study on spatial interpolation method and its application. International Conference on Audio, Language and Image Processing, 1057-1061. doi: 10.1109/ICALIP.2012.6376772.

This article accompanies the following material:

HTML:

DOI: 10.22201/igg.terradigitalis.2017.2.25.77

Static map:
DOI: 10.22201/igg.terradigitalis.2017.2.25.82 